\title{
Utilisation des marqueurs moléculaires dans les programmes d'amélioration génétique des arbres forestiers : exemple du pin maritime et de l'eucalyptus
}

\author{
C Plomion ${ }^{1}$, CÉ Durel 1, D Verhaegen 1,2 \\ 1 Laboratoire de génétique et amélioration des arbres forestiers, Inra, BP 45, 33610 Cestas ; \\ 2 Programme Plantations, Cirad Forêt, Baillarguet, BP 5035, \\ 34032 Montpellier cedex 1 , France
}

(Reçu le 19 décembre 1994 ; accepté le 27 juin 1995)

\begin{abstract}
Résumé - Les arguments émis contre l'application de la sélection assistée par marqueurs (SAM) chez les arbres forestiers, notamment les problèmes potentiels liés au manque de déséquilibre de liaison entre locus dans les populations panmictiques, sont reconsidérés en raison de la baisse des coûts et surtout de l'automatisation des techniques de marquage moléculaire issues de la technique RAPD (random amplified polymorphic DNA). La construction de cartes génétiques saturées pour les individus d'une population d'amélioration élite devrait permettre de contourner ce problème. Cette proposition est présentée et discutée. Les stratégies de cartographie génétique et de détection des facteurs génétiques contrôlant les caractères quantitatifs (QTL) s'appuyant sur des pedigrees couramment rencontrés dans les programmes d'amélioration forestiers (familles de demi-frères et de plein-frères) sont envisagées. Afin d'illustrer la faisabilité de la sélection assistée par marqueurs, des propositions sont faites pour les programmes de sélection du pin maritime et de l'eucalyptus. Les gains génétiques et le coût de la SAM sont évalués dans des cas concrets et comparés à d'autres stratégies permettant une efficacité de la sélection identique.
\end{abstract}

arbre forestier / sélection intrafamille assistée par marqueurs / multiplication végétative / marqueurs moléculaires / QTL / Pinus pinaster / Eucalyptus

Summary - Marker-assisted selection in forest tree breeding programs as illustrated by two examples: maritime pine and eucalyptus. The arguments raised against the feasibility of marker-assisted selection (MAS) in forest trees, especially the expected absence of linkage disequilibrium between markers and quantitative trait loci (QTL) in large random mating populations, are reconsidered. A decrease in costs and automation of the random amplified polymorphic DNAs (RAPD) technique make it possible to construct single-tree maps for every individual of an elite breeding population: an extreme alternative to dealing with linkage equilibrium. Mapping strategies and QTL detection using existing pedigrees in forestry breeding programs (half-sib and full-sib progenies) are presented and discussed. The feasibility of MAS is illustrated for the maritime pine and eucalyptus breeding program. Genetic gains and costs associated with the use of molecular 
forest trees / within-family marker-assisted selection / vegetative propagation / molecular markers / QTL / Pinus pinaster / Eucalyptus

\section{INTRODUCTION}

L'utilisation des marqueurs moléculaires en sélection repose sur l'exploitation des déséquilibres de liaison entre marqueurs et gènes ou QTL (quantitative trait locl) impliqués dans la variation des caractères quantitatifs. Deux stratégies d'intégration des marqueurs dans les programmes de sélection des plantes annuelles ont été envisagées.

La première vise à exploiter le déséquilibre de liaison dû à des liaisons physiques pour fabriquer un « idéotype ", individu cumulant le maximum d'allèles favorables pour des caractères d'intérêt économique. La stratégie consiste à cartographier des QTL sur une carte génétique (ex: Paterson et al, 1988) et à utiliser les marqueurs bordant pour contrôler le transfert des zones chromosomiques intéressantes. La construction d'un tel génotype peut être réalisée par backcross lorsqu'il s'agit d'intégrer dans un fond génétique préexistant un ou quelques gènes. Les marqueurs moléculaires permettent notamment de repérer les individus ayant recombiné le plus près du gène à transférer dans le parent récurrent et qui portent le moins possible d'ADN du parent donneur (Young et Tanksley, 1989 ; Ragot et al, 1995). Ils permettent également un retour plus rapide vers le génome du parent récurrent par rapport à une simple sélection basée sur l'information phénotypique (Hospital et al, 1992). Les bons allèles peuvent aussi être accumulés dans un génotype nouveau par croisement d'individus complémentaires aux QTL (Stuber et Sisco, 1993). Le nombre de croisements nécessaires pour obtenir le génotype transgressif intéressant dépendra du nombre et de la répartition des QTL dans le génome des géniteurs. Par ailleurs, plus le nombre de descendants d'un croisement donné sera important, plus la probabilité de trouver le génotype idéal sera élevée.

La deuxième stratégie vise à exploiter tous les types de déséquilibre de liaison entre marqueurs et QTL (qu'ils soient ou non dus à une liaison physique) pour évaluer la valeur génétique des individus candidats à la sélection. Dans ce cas, la position des QTL sur une carte génétique n'a pas à être connue. Les marqueurs sont traités comme des caractères associés au phénotype et en tant que tels ils doivent permettre d'augmenter l'efficacité de la sélection. Stuber et al (1982) ont montré que cette approche était possible chez le maïs et qu'en dépit du nombre important de générations de panmixie qu'avaient subi les populations un déséquilibre de liaison subsistait et permettait d'obtenir des associations significatives entre marqueurs et QTL de caractères agronomiques. Lande et Thompson (1990) ont posé les bases théoriques de la sélection assistée par marqueurs (SAM) en intégrant les données moléculaires dans un index de sélection.

Étant donné les caractéristiques des arbres forestiers (longévité, diversité génétique élevée et population d'amélioration en équilibre de liaison), peut-on envisager chez ces espèces de telles applications avec les types de familles généralement mises en place dans les programmes de sélection (familles de demi-frères et familles de plein-frères) ? L'objectif de cet article est d'apporter des éléments de réponse à cette question, d'estimer les gains génétiques et les coûts de la sélection assistée par marqueurs. Nous proposons et 
discutons tout d'abord une stratégie générale de sélection intrafamille assistée par marqueurs, basée sur un argumentaire technique et génétique. Nous envisageons ensuite l'application de cette stratégie dans les programmes de sélection du pin maritime et de l'eucalyptus, deux espèces qui font l'objet d'un programme d'amélioration génétique et d'un important effort de création variétale.

\section{Caractéristiques des espèces d'arbres forestiers et proposition d'une stratégie de sélection assistée par marqueurs}

Les cycles de sélection sont généralement longs chez la plupart des espèces forestières. Ce sont surtout les délais nécessaires à l'évaluation des génotypes et/ou les délais de maturité sexuelle qui constituent les facteurs limitants à l'amélioration génétique. Par exemple, pour qu'une sélection efficace puisse être effectuée, il faut attendre presque un tiers de l'âge de la rotation, soit 10 à 15 ans chez le pin maritime, (Kremer 1992 ; Danjon, 1994), 6 à 8 ans chez le pin taeda (McKeand, 1988) et le pin radiata (Cotterill et Dean, 1988), 2 à 3 ans chez l'eucalyptus (Bouvet et Vigneron, 1995). De plus, les délais nécessaires jusqu'à la première fructification entraînent une évaluation des principaux caractères d'intérêt économique (vigueur, forme, qualité du bois) à partir d'un certain âge : plus de 10 ans pour la plupart des conifères et plus de 3 ans chez les hybrides interspécifiques d'eucalyptus (Bouvet, 1991). Dès lors, il apparaît clairement que la première approche : construction rapide d'un « idéotype " par backcross ou sélection généalogique assistée par marqueurs, est difficilement envisageable.

Les arbres forestiers sont généralement des espèces allogames peu domestiquées, caractérisées par un haut niveau de diversité génétique (Hamrick et al, 1992) et un faible déséquilibre de liaison entre mar- queurs et QTL (revu par Strauss et al, 1992). Ce dernier point constitue certainement la limitation majeure à l'utilisation des marqueurs moléculaires dans le cadre de la seconde approche (Avery et Hill, 1979 ; Muona, 1982 ; Beckman et Soller, 1983 ; Beckman et Soller, 1986 ; Hasting 1989 ; Lande et Thompson, 1990). En effet, selon la théorie de la génétique des populations, dans les populations à régime de reproduction allogame, les allèles au marqueur et au QTL seraient associés au hasard d'un arbre à l'autre. II serait donc impossible d'établir de façon significative une association marqueur-QTL au niveau de la population. De plus, le soin pris pour éviter les croisements entre apparentés dans la plupart des populations d'amélioration restreint d'autant la probabilité de créer tout déséquilibre de liaison. Là encore, l'utilisation des marqueurs comme caractères associés pour mieux estimer la valeur génétique des individus apparaît limitée.

Cependant, les avancées récentes des techniques de marquage moléculaire permettent d'envisager une stratégie intermédiaire : la sélection intrafamille assistée par marqueur. Un index de sélection combinant l'information des caractères phénotypiques et des marqueurs bordant les QTL détectés pourrait être utilisé pour rechercher les meilleurs individus dans chacune des meilleures familles. Un tel index basé sur la position la plus probable des QTL potentiels est plus puissant que celui proposé par Lande et Thompson (1990) car il prend en compte la recombinaison entre marqueurs et QTL (B Goffinet, communication personnelle). Cette proposition nécessite la construction de cartes génétiques et la localisation de QTL pour chaque famille. Étant donné les coûts d'une telle opération (discutés ultérieurement), il est évident que notre proposition ne pourra pas s'appliquer à l'ensemble d'une population d'amélioration : à titre d'exemple, la population d'amélioration « G1 » de deuxième génération du pin maritime contient 1100 individus. 
Au contraire, l'utilisation d'une nouvelle technologie de sélection utilisant les marqueurs de l'ADN pourrait être appliquée dans le cadre d'une stratégie de sélection intense et à court terme, utilisant un sousensemble de géniteurs "élites " aux performances connues. Cette solution, qui exploite les déséquilibres de liaison physiques, ne pouvait s'envisager pratiquement que par rapport à l'automatisation et à la baisse des coûts des techniques de marquage moléculaire. Nous verrons dans un premier temps comment les marqueurs RAPD (Random amplified polymorphic DNA, Williams et al, 1990) dérivés de la PCR (Polymerase chain reaction, Mullis et Faloona, 1987) offrent cette potentialité. Nous développerons ensuite les arguments génétiques de la sélection assistée par marqueurs (SAM) intrafamille. Finalement, nous discuterons de l'utilisation des marqueurs dans les programmes de sélection du pin maritime et de l'eucalyptus.

\section{Argument technique : un procédé de marquage simple, rapide et peu coûteux}

La SAM intrafamille nécessite une technique de marquage efficace permettant de génotyper rapidement avec un faible coût un grand nombre de descendants des familles élites (l'effectif sera discuté au paragraphe « Effectifs nécessaires pour la SAM intrafamille "). Nous avons envisagé, pour cinq systèmes de marquage moléculaire, les principales caractéristiques génétiques et techniques ainsi que les points forts et les points faibles de certains critères d'utilisation en sélection (tableau I). Les systèmes isoenzymatiques sont très faciles et peu coûteux à révéler. Cependant, le nombre de marqueurs révélés par cette technique reste trop faible pour des applications qui nécessitent une saturation du génome, telles que la cartographie de locus contrôlant des caractères quantitatifs. Les protéines révélées par électrophorèse bidi- mensionnelle représentent une technique de marquage difficile à mettre en œuvre et à interpréter à grande échelle. Les marqueurs codominants dérivés de la PCR (ex : les microsatellites) sont encore trop peu développés chez les arbres forestiers (Smith et Devey, 1994) pour envisager leur utilisation à court terme dans un programme de sélection. De plus, le peu de microsatellites obtenus chez les gymnospermes se sont révélés monomorphes (Hutchison et al, 1994). Les conifères présentent un taux de recombinaison par nucléotide très faible car leur génome est de très grande taille. Or, les microsatellites sont généralement générés lors de la méiose par des crossing-over inégaux. Une fréquence chiasmatique faible, relativement au nombre de nucléotides, pourrait expliquer la difficulté de trouver des microsatellites polymorphes chez la plupart des conifères. Les marqueurs RFLPs (Restriction fragment length polymorphism, Botstein et al, 1980) sont intéressants à plusieurs titres (codominance, multiallélisme, potentialité d'utilisation chez d'autres espèces du même genre). Cependant, la durée de mise en œuvre de cette technique peut prendre plusieurs années chez les espèces où des banques génomiques et des sondes moléculaires n'ont pas encore été développées. C'est le cas pour la majorité des espèces forestières. D'autre part, chez certaines espèces, comme les Pinus, la quantité d'ADN est si élevée (15 à $30 \mathrm{pg} / \mathrm{C}$, Ohri et Khoshoo, 1986 ; Wakamiya et al, 1993) qu'il est difficile de trouver des sondes "simple-copies ", c'est-à-dire s'hybridant sur une zone unique du génome. L'utilisation de la radioactivité et des temps d'exposition très longs constituent une limite supplémentaire à l'utilisation des marqueurs RFLP dans un programme de sélection assistée par marqueurs chez ces espèces.

Au contraire, la technique RAPD est simple, accessible à tous et extrêmement efficace en termes de temps d'acquisition des 
données (Kesseli et al, 1994 ; Nelson et al., 1995). De plus, les marqueurs RAPD sont très polymorphes chez les espèces allogames et leur nombre est illimité (tableau I). Par ailleurs, leur dominance (Williams et al, 1990 ; Williams et al, 1993) n'est pas un handicap pour les stratégies de cartographie développées chez les arbres forestiers (voir paragraphe « Des stratégies de cartographie efficaces "). Nous estimons qu'à raison de 400 réactions d'amplification PCR par jour la recherche préalable de polymorphisme ainsi que l'établissement d'une carte génétique peut être réalisée en routine en deux mois et ce, par une seule personne. En moyenne, trois marqueurs polymorphes sont obtenus par sonde oligonucléotidique (Grattapaglia et Sederoff, 1994 ; Plomion et al, 1995a). La construction d'une carte saturée peut être entreprise en génotypant une soixantaine d'individus avec une centaine d'amorces oligonucléotidiques de dix paires de bases. Les coûts de réalisation d'une carte à l'aide de tels marqueurs sont relativement faibles (tableau II). Environ $80 \%$ du coût est imputable au prix commercial de la Taq polymérase (enzyme utilisé à raison d'une unité par réaction), les $20 \%$ restant sont répartis entre le prix des oligonucléotides, des nucléotides tri-phosphates A (adénine), $T$ (thymine), $C$ (cytosine) et $G$ (guanine), de l'agarose et de divers tampons de réaction et de migration. L'utilisation d'imprimantes thermiques permet de limiter considérablement le prix des photographies. Le prix d'une unité de Taq polymérase est d'environ $1 \mathrm{~F}$.

Les rares études comparant les coûts des techniques de marquage RFLP et RAPD (marqueurs les plus utilisés pour la cartographie génétique chez les espèces végétales) montrent un léger avantage à la première (ex : Ragot et Hoisington, 1993). Cependant, ces études sont basées sur des espèces chez lesquelles plusieurs centaines de clones d'ADNc ou d'ADN génomique sont déjà disponibles, comme chez le mais (Gradiner et al, 1993). Or, chez la plupart des espèces d'arbres forestiers, pratiquement aucune information génomique n'est actuellement disponible (banque d'ADN ou de d'ADNc, sondes moléculaires, séquences géniques). Un nombre limité de sondes ont été développées récemment chez le pin taeda (Devey et al, 1994), le peuplier (Bradshaw et al, 1994) et l'eucalyptus (Byrne et al, 1995). La mise en œuvre de la technique RAPD, qui ne nécessite aucune connaissance a priori de séquences de l'ADN, offre ainsi la possibilité de générer des cartes génétiques rapidement (Nelson et al, 1994) et à faible coût chez de nombreuses espèces végétales.

\section{Arguments génétiques et contraintes}

\section{Des stratégies de cartographie efficaces}

Les populations généralement utilisées pour la réalisation de cartes génétiques chez les espèces végétales annuelles : F2, back-cross, lignées recombinantes (Stuber, 1992), ne sont pas disponibles chez la plupart des espèces forestières, à cause du temps de génération et de la dépression de consanguinité quasi générale (Sorensen et Miles, 1982 ; Griffing et Cotterill, 1988 ; Sniezko et Zobel, 1988). Les stratégies de cartographie génétique utilisant des marqueurs dominants (exemple: RAPD) qui ont été développées s'appuient sur les deux types de familles les plus couramment rencontrées dans les programmes d'amélioration des arbres forestiers : descendances de demi-frères issues de pollinisation libre ou contrôlée et descendances de plein-frères.

Cartographie génétique et détection de QTL dans une famille de demi-frères, utilisant des marqueurs RAPD

Chez les espèces allogames, la cartographie génétique ainsi que la détection de QTL à partir d'une famille de demi-frères (famille HS) dépend essentiellement de la 


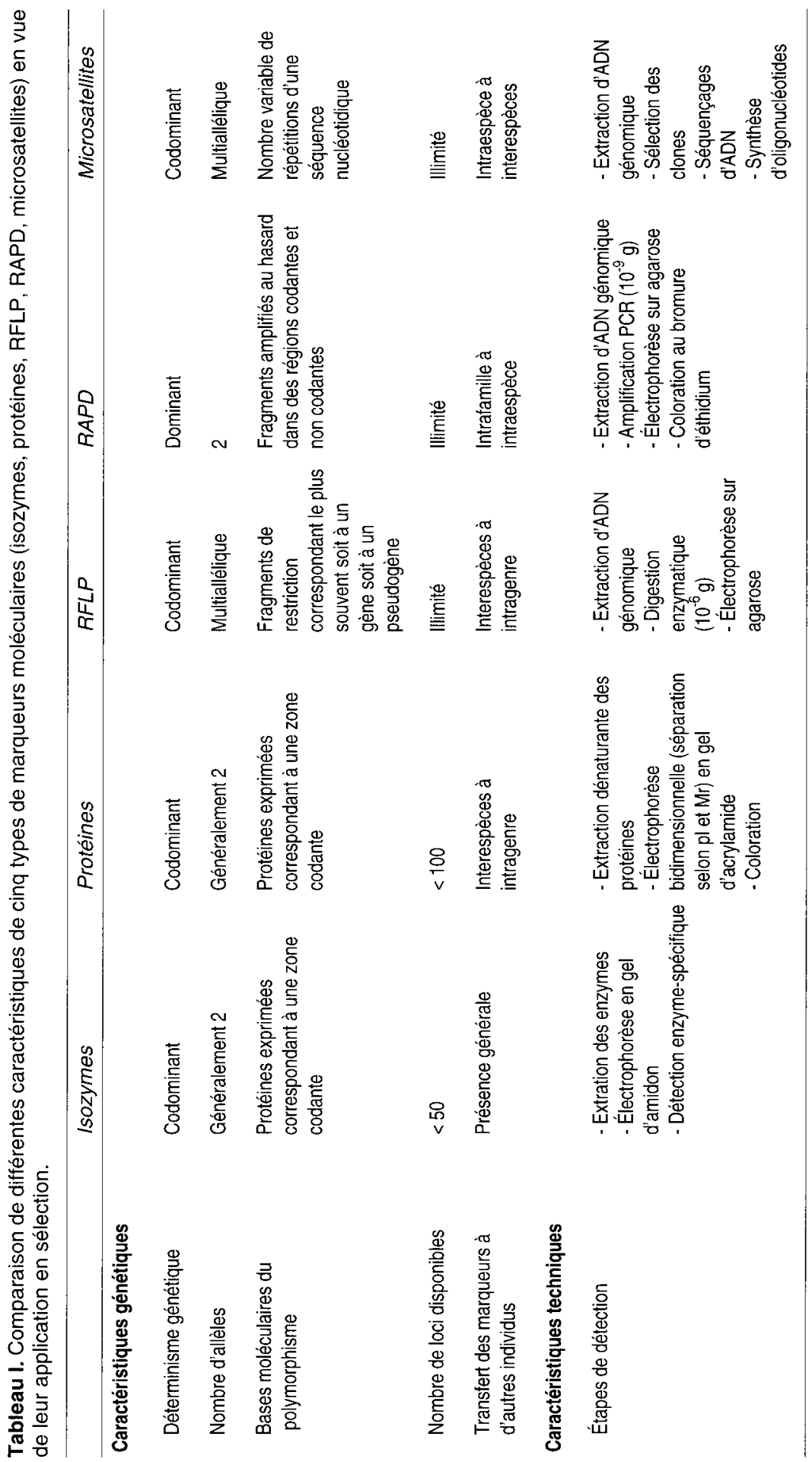




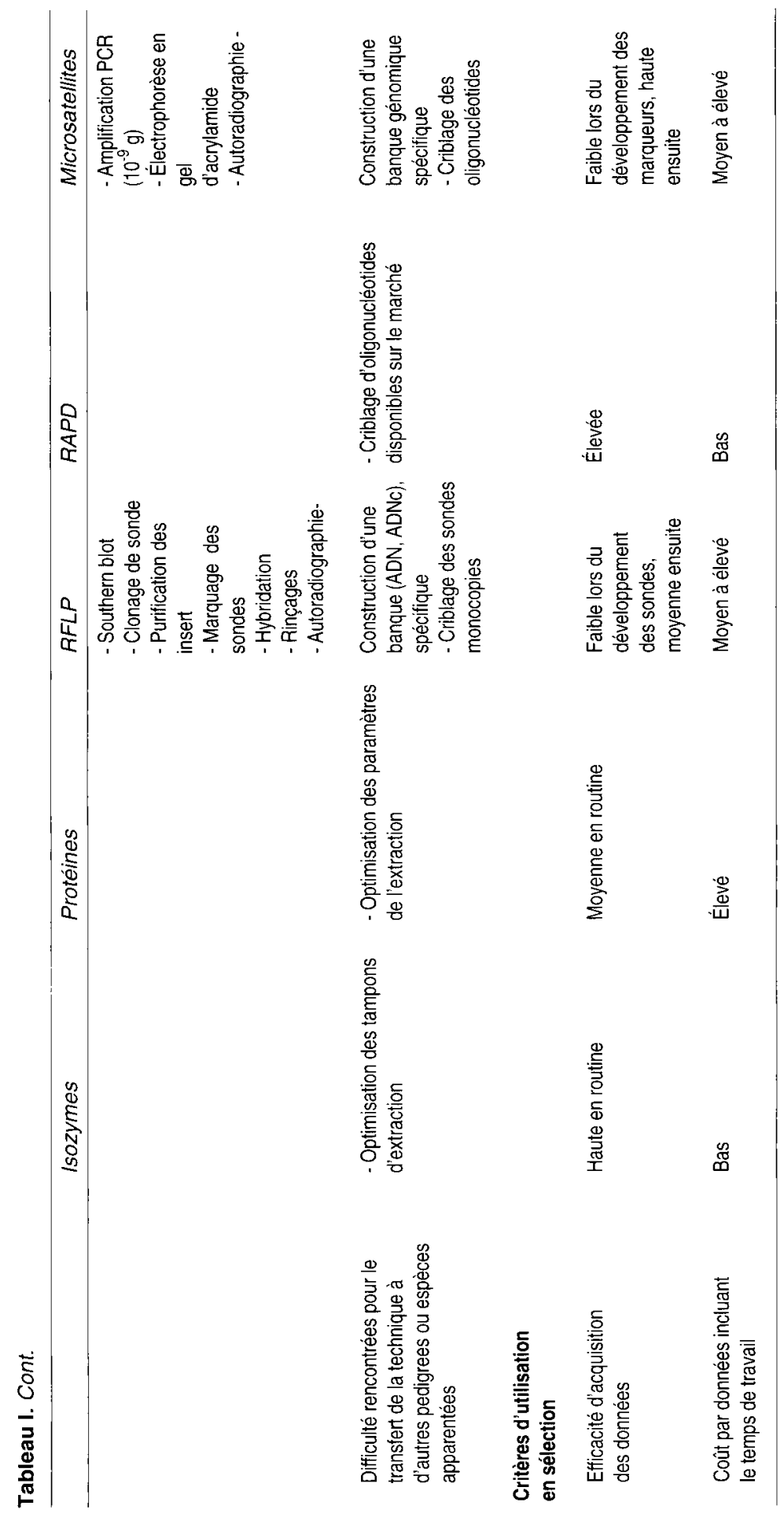




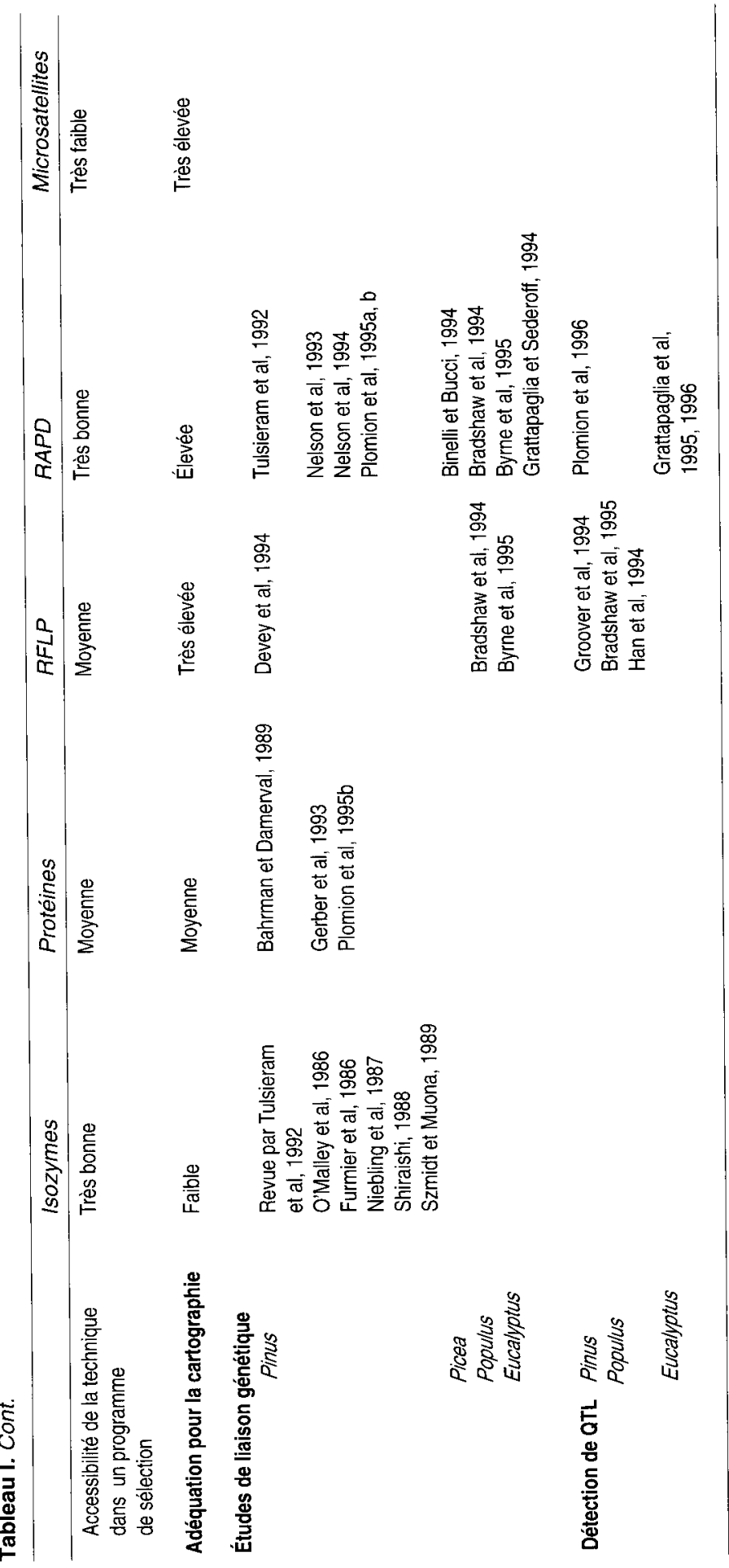


possibilité de distinguer la contribution allélique maternelle dans la descendance.

Chez les végétaux, la cartographie utilisant des familles HS a d'abord été appliquée au mégagamétophyte des gymnospermes (Conkle, 1981), tissu de réserve haploïde d'origine maternelle, dérivant par divisions méiotiques puis mitotiques de la mégaspore. Chaque mégagamétophyte d'un arbre porte l'information génétique d'un unique événement de la méiose. Embryon et mégagamétophyte d'une même graine diffèrent donc par la contribution gamétique paternelle. Ainsi, les marqueurs révélés à partir des mégagamétophytes d'un même arbre montrent un ratio de ségrégation 1:1. La stratégie de cartographie consiste à examiner la ségrégation d'un grand nombre de marqueurs sur un échantillon de 60 à 100 mégagamétophytes extraits des graines d'un même arbre. Des pedigrees étendus ne sont pas nécessaires et n'importe quel arbre sexuellement mature peut être cartographié. Les premières cartes de liaison ont été établies à partir de ce tissu chez diverses espèces de pins pour des marqueurs enzymatiques et protéiques (tableau 1). La faible quantité d'ADN extraite d'un mégagamétophyte (de 1 à $2 \mu \mathrm{g}$ ), rend impossible la révélation de marqueurs RFLP qui nécessite une dizaine de microgrammes d'ADN pour une hybridation sur un génome aussi grand que celui des conifères (Devey et al, 1994). Inversement, cette quantité d'ADN permet de réaliser des centaines de réactions PCR et

Tableau II. Coût de la cartographie et de la détection de QTL spécifiques et généraux, à partir d'une famille de plein-frères de 200 individus et de deux familles de demi-frères de 200 individus.

\begin{tabular}{lccc}
\hline Étapes & $\begin{array}{c}\text { Nombre de } \\
\text { réactions }\end{array}$ & & Coûts \\
\cline { 3 - 4 } & & Fonctionnement Main-d'oeuvre $^{\mathrm{b}}$ \\
$\begin{array}{l}1-\text { Extraction d'ADN } \\
(600 \text { individus par la méthode Doyle et Doyle, } \\
1987)\end{array}$ & 600 & $600 \mathrm{~F}$ & $10 \mathrm{TJ}=10$ \\
\end{tabular}

\section{2 - Cartographie}

2.1 - Criblage des marqueurs polymorphes (six individus plein-frères et deux parents $\times 500$ amorces)

2.2 - Génotypage

(60 individus plein-frères $X 100$ amorces)

3 - Détection de QTL spécifiques

(140 individus plein-frères $X 40$ amorces)

4 - Détection de QTL généraux

(400 individus demi-frères $X 15$ amorces)

\begin{tabular}{ccc}
4000 & $4800 \mathrm{~F}$ & $\begin{array}{c}10 \mathrm{TJ}=10 \\
000 \mathrm{~F}\end{array}$ \\
6000 & $7200 \mathrm{~F}$ & $\begin{array}{c}15 \mathrm{TJ}=15 \\
000 \mathrm{~F}\end{array}$ \\
5600 & $6720 \mathrm{~F}$ & $\begin{array}{c}14 \mathrm{TJ}=14 \\
000 \mathrm{~F}\end{array}$ \\
6000 & $7200 \mathrm{~F}$ & $\begin{array}{c}15 \mathrm{TJ}=15 \\
000 \mathrm{~F}\end{array}$ \\
& & $\begin{array}{c}64 \mathrm{TJ}=64 \\
\text { SOUS-TOTAL }\end{array}$ \\
$\begin{array}{c}26520 \mathrm{~F} \\
\text { TOTAL }\end{array}$ & $90520 \mathrm{~F}$ & $000 \mathrm{~F}$ \\
\hline
\end{tabular}

${ }^{a}$ Coût d'une réaction RAPD $=1,20 \mathrm{~F}$, coût d'une extraction d'ADN $=1,0 \mathrm{~F} ;{ }^{b} 1 \mathrm{TJ}$ (un technicien jour $=1000 \mathrm{~F}$ TTC)

$=60$ extractions d'ADN, 400 réactions PCR (réaction, migration, photographie, saisie des données). 
ainsi de produire de nombreux marqueurs RAPD directement utilisables pour la cartographie génétique et la détection de QTL (tableau I). Le déterminisme génétique essentiellement dominant des marqueurs RAPD n'est pas limitant pour cette stratégie de cartographie équivalente à la stratégie appliquée à une famille issue d'un testcross. L'utilisation de la phase gamétophytique est très pratique pour la réalisation de cartes génétiques. Cependant, le mégagamétophyte est un tissu “ temporaire " que l'on ne peut prélever qu'au moment de la germination des plants. Son utilisation restreint donc la cartographie de QTL à des familles jeunes spécialement mises en place dans ce but. L'utilisation de la phase sporophytique est cependant essentielle car le génotypage d'individus adultes existant dans les tests de descendances permettra d'utiliser directement l'information des marqueurs pour la sélection de caractères d'intérêt économique.

La cartographie de marqueurs RAPD peut être réalisée à partir d'une famille de demi-frères sur matériel diploïde. Cette stratégie également analogue à un testcross est applicable chez les gymnospermes comme chez les angiospermes. Elle est basée sur l'hypothèse de l'existence d'allèles rares présents à l'état hétérozygote chez la mère et à une très faible fréquence, voir nulle dans le mélange pollinique. Les marqueurs ayant de tels allèles ségrégeront par conséquent de façon quasi mendélienne dans la descendance de demi-frères, dans des proportions voisines du ratio 1:1. La technique RAPD très sensible au changement d'un seul nucléotide offre la possibilité de rechercher rapidement de nombreux marqueurs informatifs vérifiant la présence d'allèles rares chez la mère et permettant aussi l'identification de cet allèle maternel dans la descendance (Grattapaglia et al, 1996). Paradoxalement, le fait que les marqueurs RAPD soient dominants, et ne détectent donc qu'un seul allèle à un locus, facilite l'apparition de telles configurations, car le génotype nul des pères correspond à des allèles non détectés au locus RAPD. Des simulations réalisées avec des marqueurs dominants et codominants ont montré que, pour des allèles ayant une fréquence inférieure ou égale à 0,2 dans le mélange pollinique, les marqueurs présentent des distortions de ségrégation compatibles avec la cartographie de marqueurs et de QTL (Liu et al, 1993). Chez les animaux une approche identique a été développée avec des marqueurs microsatellites (Haley, 1991).

Pour l'analyse des QTL, seule la contribution femelle de la descendance de demifrères est prise en compte. La coségrégation entre marqueurs et caractères quantitatifs aboutit à la détection de QTL dans un fond génétique constitué d'un mélange de parents mâles. Cette stratégie a été utilisée chez les animaux par Beever et al (1990) et chez les plantes par Grattapaglia et al (1996). Soit un QTL multiallélique (Qi), la valeur du QTL associée aux allèles maternels des deux génotypes au marqueur représente un effet de substitution allélique (Q1 par rapport à $Q 2)$ moyenné sur tous les allèles $\left(Q_{j}\right)$ du pool pollinique. Cette stratégie permet de détecter des QTL qui ont une valeur relative à la population de référence qui a servi pour le polycross. Le choix du polycross déterminera la généralisation de ces QTL à l'ensemble de la population. L'analyse aboutit à l'estimation de la valeur d'aptitude générale à la combinaison (effet additif) des allèles au QTL pour le parent femelle.

La principale limitation de cette technique reste le nombre de demi-frères à génotyper pour atteindre une puissance de détection de QTL satisfaisante. En utilisant la méthode du maximum de vraisemblance, $\mathrm{Ha}$ ley (1991) estime qu'une puissance statistique de 0,67 devrait être atteinte avec un effectif de $n=400$ individus, une erreur de type I $\alpha=0,001$, un écart phénotypique 0,5 $\sigma$ entre les deux groupes de demi-frères portant 
des allèles alternatifs au marqueur et une distance $\theta=10 \mathrm{cM}$ entre le marqueur et le QTL. Pour un effet de $0,25 \sigma$, une puissance maximale de 0,24 serait atteinte avec une densité infinie de marqueurs $(\theta=0 \mathrm{cM})$. Weller et al (1990) ont pour leur part estimé que la détection de QTL ayant un effet de 0,3 $\sigma$ nécessitait de 1000 à 2000 descendants. Ils ont proposé une stratégie de détection de QTL chez les animaux, basée sur des pedigrees à trois générations, et ont montré qu'une puissance satisfaisante pouvait être atteinte avec un effectif moindre. Cependant, chez les arbres forestiers les pedigrees à trois générations sont rares. La sélection est basée sur des pedigrees à deux générations avec des effectifs généralement inférieurs à 100 individus. Ainsi, la détection de QTL à partir d'une famille de demi-frères apparaît limitée car elle nécessiterait des effectifs importants (> 500 individus), rarement rencontrés dans les familles mises en test.

\section{Cartographie génétique et détection de} QTL dans une famille de plein-frères, utilisant des marqueurs RAPD

Le principe de la cartographie de marqueurs RAPD à partir d'une descendance de plein-frères est le suivant : si l'un des parents d'un croisement contrôlé est hétérozygote pour un marqueur donné alors que l'autre parent est homozygote nul pour ce même marqueur, la descendance hybride ségrégera comme dans un testcross, dans les proportions mendéliennes 1:1 pour un tel marqueur (Carlson et al, 1991). Cette stratégie peut s'appliquer simultanément à chacun des deux parents, elle est appelée «double pseudo-testcross 》 (Grattapaglia et Sederoff, 1994 ; Echt et al, 1994). L'avantage des marqueurs RAPD réside dans l'opportunité de cribler une quantité importante d'amorces oligonucléotidiques pour révéler de tels marqueurs informatifs. Le niveau d'hétérozygotie généralement très élevé chez la plupart des espèces forestières permet de retrouver maintes fois de telles configurations. Les mêmes amorces oligonucléotidiques permettent de réveler du polymorphisme chez les deux parents et, finalement, deux cartes génétiques distinctes pour les deux parents peuvent être établies. La fusion des deux cartes peut être envisagée lorsque des marqueurs hétérozygotes ont été identifiés chez les deux parents. Ces marqueurs ségrègent dans les proportions mendéliennes 3:1 dans la famille hybride et peuvent servir de jalons communs pour l'établissement d'une carte consensus (Cai et al, 1994). Cependant, la fonction d'information (Allard, 1956) entre couples de marqueurs ségrégeant dans des proportions 1:1 et 3:1 est faible (Ritter et al, 1990). Si la fusion des deux cartes est vraiment recherchée l'utilisation de marqueurs multialléliques (type RFLP ou microsatellites) ségrégeant 1:1:1:1 dans la descendance hybride sera plus puissante (van Eck et al, 1994).

L'analyse des QTL par la stratégie du “ double pseudo-testcross " est également analogue à l'analyse des QTL dans un testcross. Deux analyses sont réalisées séparément pour chacun des deux parents et des QTL spécifiques, ayant une valeur relative au croisement réalisé, peuvent être détectés (Grattapaglia et al, 1995). L'effet de substitution allélique (modification de la valeur du génotype entraînée par le remplacement d'un allèle par un autre, face à des allèles inconnus) est alors déterminé pour chaque QTL. II inclut des effets additifs et de dominance, sur la base du modèle génétique (Leonards-Schippers et al, 1994). En effet, soit un QTL multiallélique $\left(Q, Q_{j}\right)$ lié à un marqueur RAPD $(\mathrm{Mm})$; si le génotype au QTL lié au marqueur est $Q_{1} Q_{2}$ chez un parent et $Q_{3} Q_{4}$ chez l'autre parent, on testera la différence entre la valeur moyenne du caractère des individus $\left[Q_{1} Q_{3}+Q_{1} Q_{4}\right]$ portant l'allèle $M$ au marqueur, par rapport à la valeur moyenne du caractère des individus $\left[Q_{2} Q_{3}+Q_{2} Q_{4}\right]$ por- 
teur de l'allèle nul, m, au marqueur ; et vice versa pour l'autre parent.

Les deux stratégies que nous venons de décrire s'appuient sur des pedigrees à deux générations et sont applicables dans la plupart des programmes de sélection. Cependant, la SAM intrafamille s'oriente plutôt vers l'utilisation de familles de pleinfrères. En effet, d'une part les QTL sont statistiquement plus faciles à détecter que dans des familles de demi-frères car ils incluent des composantes additive et de dominance, et d'autre part la stratégie « double-pseudo testcross " ne nécessite pas le criblage systématique d'allèles rares. L'identification des QTL spécifiques constitue une première étape de la SAM intrafamille de plein-frères. Nous verrons dans le paragraphe "Recherche des génotypes transgressifs dans des familles de plein-frères, combinée à la multiplication clonale " que ces QTL spécifiques peuvent être valorisés directement dans le cadre de la création de variétés clonales. Cependant, l'utilisation des QTL spécifiques dans un programme de sélection nécessitera de tester dans un deuxième temps leur valeur générale sur la population d'amélioration. Nous verrons comment ceci est possible en utilisant des familles polycross dans le paragraphe "Méthodologie de la sélection intrafamille assistée par marqueurs".

\section{Des caractères cibles à héritabilité faible à moyenne}

La sélection des individus candidats à la sélection se fait généralement de façon multicaractère par le calcul d'un index de sélection combiné «individu-famille ". Dans le cas du pin maritime, environ la moitié des familles sont retenues et les trois à cinq meilleurs index par famille sont sélectionnés de manière à conserver une variabilité génétique suffisante. La plupart des caractères économiquement importants pris en compte dans les index de sélection sont des caractères complexes à héritabilité (sensus stricto) faible à moyenne (Zo- bel et Talbert, 1984 ; Cornelius, 1993 ; Kremer, 1994), généralement variant de 0,10 à 0,40 . Le déterminisme génétique de ces caractères est souvent considéré comme polygénique avec un fort effet du milieu. Le choix des meilleurs index intrafamille ne garantit donc pas la sélection des meilleurs individus à l'intérieur de ces familles. Des méthodes visant à améliorer l'efficacité de cette sélection intrafamille sont donc à rechercher. L'évaluation plus précise des génotypes peut faire appel à l'étude des effets microenvironnementaux (Pichot, 1993), et des effets de compétition sur l'expression des caractères. D'autre part, les résultats récents provenant principalement d'études menées sur les espèces de grande culture suggèrent que les caractères quantitatifs seraient principalement contrôlés par peu de gènes (revu par Stuber, 1992 et Dudley, 1993), et que ces gènes n'ont pas un effet de même amplitude. Les résultats expérimentaux obtenus mettent en évidence une distribution " en L » de l'effet des QTL (peu de gènes à effets forts et beaucoup de gènes à effets faibles, Shrimpton et Robertson, 1988). Des méthodes de sélection basées sur le calcul d'indices de sélection prenant en compte les marqueurs ont été récemment développées (Liu et Hayes, 1993 ; Liu et al, 1994). Elles tiennent compte des acquis des dernières années concernant le contrôle génétique des caractères quantitatifs. Des simulations montrent que, pour toute une série de conditions expérimentales (densité de la carte génétique, héritabilité du caractère, intensité de sélection, nombre et effet de chaque QTL, taille de la population), la SAM est plus efficace que la sélection basée sur la seule information du phénotype (BH Liu, communication personnelle). Lorsque l'héritabilité au sens strict est comprise entre 0,2 et 0,4 , ce qui est fréquemment le cas chez les espèces forestières surtout pour les caractères de croissance (Cornelius, 1993), une sélection combinant l'information phénotypique et marqueurs molécu- 
laires devrait être efficace (Stuber, 1989 ; Paterson et al, 1991 ; Lande et Thompson, 1990 ; Strauss et al, 1992), surtout dans le cas d'une sélection multicaractère. Cependant, pour des héritabilités trop faibles, l'efficacité de la SAM peut devenir comparable à celle de la sélection basée sur le phénotype du fait de la mauvaise précision de l'estimation des effets aux QTL.

\section{Effectifs nécessaires pour la SAM intrafamille}

Notre objectif principal est d'utiliser les marqueurs pour la sélection intrafamille, sur des pedigrees adultes actuellement en place dans les tests de descendances. Or, la puissance de détection de QTL dépend surtout du nombre d'individus génotypés, du type de population utilisée, de la précision apportée aux mesures des caractères quantitatifs et de l'effet des QTL qui ségrègent dans la population. En revanche, elle est faiblement fonction de la densité de marquage de la carte génétique (Darvasi et al, 1993). Il apparaît donc clairement qu'un des premiers critères d'application de la SAM intrafamille concerne l'effectif des descendances présentes sur le terrain. Strauss et al (1992) ont estimé qu'environ 200 individus plein-frères permettaient de détecter la moitié de la variance additive avec une erreur de type I de $1 \%$ lorsque l'héritabilité (sensu stricto) du caractère est de 0,5 et que cinq QTL contrôlent le caractère quantitatif. Des héritabilités de 0,5 sont rarement rencontrées (sauf par exemple pour l'infradensité du bois, Cornelius, 1993). Ainsi, un effectif de 200 individus doit être considéré comme une limite inférieure sauf dans le cas où l'on dispose de copies clonales des individus. Bradshaw et Foster (1992) ont montré l'intérêt d'utiliser de telles répétitions clonales surtout lorsque l'héritabilité du caractère est faible. Knapp et Bridges (1990) ont par ailleurs montré que, si toute la variance génétique additive était expliquée par les marqueurs, disposer d'une seule répétition clonale per- mettait d'augmenter la puissance statistique de détection d'une quantité équivalente à celle obtenue si on avait doublé l'effectif de la population d'origine.

\section{L'instabilité des associations marqueurs-QTL : un frein potentiel à l'application de la SAM chez les arbres forestiers}

\section{Stabilité des associations marqueurs-QTL vis-à-vis du milieu}

Le manque de stabilité des associations marqueurs-QTL selon l'environnement a été observé chez les plantes annuelles de grande culture (Paterson et al, 1991 ; Hayes et al, 1993). Trois cas d'interaction QTL x milieu peuvent être rencontrés : i) QTL spécifiques à certains environnements ; ii) changement de l'effet des mêmes QTL selon le milieu (effet d'échelle) ; iii) expression opposée d'allèles favorables aux QTL dans des milieux différents. Le troisième cas correspond à un changement de rang des performances des génotypes. Il devrait constituer l'aspect le plus limitant de la SAM. Si l'importance des interactions QTL $x$ milieu était confirmée, elle obligerait à une certaine prudence dans l'utilisation des marqueurs moléculaires et notamment pour l'extrapolation de résultats obtenus en un lieu.

Cependant, il convient de noter que le problème de l'instabilité des performances prédites par les génotypes aux marqueurs n'est pas uniquement réservé à la SAM. II est en effet à prendre en considération dans toutes formes de sélection artificielle, et le développement de variétés adaptées à différents environnements entraîne toujours des coûts techniques et financiers supplémentaires.

\section{Stabilité des associations marqueurs-QTL selon le stade de développement}

L'application potentielle la plus attirante des marqueurs moléculaires concerne cer- 
tainement leur utilisation pour la sélection précoce. Or, chez les espèces longévives comme les arbres forestiers, une des causes possibles du manque de corrélation juvénile-adulte pour les caractères liés à la croissance pourrait résulter d'un changement du contrôle génétique des caractères au cours du temps (Greenwood et al, 1989 ; Kremer et al, 1991). L'étude des interactions QTL x âge est donc une étape clé pour évaluer le potentiel d'application de la sélection précoce assistée par marqueurs. À l'heure actuelle, trop peu de résultats (Plomion, 1995) permettent de donner une réponse à ce problème. L'enjeu est primordial, car des gains génétiques très importants par unité de temps pourraient en résulter.

Stabilité des associations marqueurs-QTL selon le fond génétique

Des résultats préliminaires d'études menées chez le maïs et la tomate montrent que l'expression des QTL ne serait pas constante selon les descendances dans lesquelles elle a été recherchée (Beavis et al, 1991 ; Tanksley et Hewitt, 1988 ; Graef et al, 1989 ; Stuber, 1989). II n'est pas possible de savoir a priori si un QTL détecté dans une famille particulière correspond à un événement rare ou pourra être retrouvé dans d'autres contextes génétiques (Edwards, 1992). L'importance de ce résultat est étroitement liée à la recherche et à l'exploitation en sélection de QTL identifiables chez tous les individus de la population d'amélioration. En revanche, la non stabilité des associations marqueurs-QTL selon le fond génétique n'apparaît pas comme une limitation dans la stratégie de SAM intrafamille telle que nous la développons ici.

\section{Utilisation des marqueurs moléculaires dans le programme d'amélioration du pin maritime et de l'eucalyptus}

Dans le premier exemple (pin maritime), nous envisageons l'utilisation des mar- queurs moléculaires comme information complémentaire à la mesure d'un caractère quantitatif d'intérêt économique, pour estimer la valeur génotypique des individus candidats à la sélection (Gallais, 1993). Dans le deuxième exemple (eucalyptus), nous montrons comment les marqueurs pourraient être utilisés d'une part pour obtenir une meilleure estimation de la prédiction de la vigueur hybride, et d'autre part pour sélectionner de bons génotypes dans des familles de plein-frères pour la création variétale par multiplication clonale. Dans ces deux espèces, l'utilisation des marqueurs et l'identification de QTL est envisagée pour obtenir une meilleure estimation sur la prédiction de la valeur génétique d'un individu issu d'une famille de plein-frères. Nous considérons notamment les gains génétiques et les coûts d'une telle approche.

\section{Intégration de la sélection intrafamille assistée par marqueurs dans le schéma de sélection du pin maritime}

\section{Évolution du schéma de sélection}

Le pin maritime fait actuellement l'objet d'un programme d'amélioration selon un schéma de sélection récurrente, associant l'élimination de certaines familles et la sélection des index multicaractères les plus élevés dans les meilleures familles (Durel, 1990). Au début des années 1960 , des sélections phénotypiques d'arbres «plus " en forêt ont permis de constituer la population de base (génération Go comportant 550 géniteurs) du programme d'amélioration génétique (Illy, 1966). Des tests de descendances ont alors été mis en place afin d'évaluer les performances des arbres sélectionnés et environ 1100 individus G1 constituant la deuxième population d'amélioration ont été sélectionnés au cours des années 1980. Les géniteurs G2 de troisième génération sont en cours de sélection (Durel, 1992). 
L'effectif de la population d'amélioration de deuxième génération ( 1100 individus G1) est très lourd à gérer même dans le cadre d'une coopération avec des organismes extérieurs. Par ailleurs, malgré un nombre important d'individus, la base génétique de la population $\mathrm{G} 1$ reste faible puisque seulement 350 mères $\mathrm{G} 0$ ont rééllement été impliquées dans les croisements. Il y a donc des risques certains d'apparentement dans les générations suivantes si l'on poursuit le schéma récurrent. À court terme, il est donc souhaitable que le schéma de sélection soit réorganisé.

L'utilisation de populations élites composées de quelques individus très sélectionnés alimentées régulièrement par la population d'amélioration principale, elle-même subdivisée en sous-populations non apparentées, a récemment été proposé pour restructurer les programmes de sélection d'arbres forestiers les plus avancés (McKeand et Bridgwater, 1992; White et al, 1993). Les populations élites ainsi que les meilleurs individus de la population principale font l'objet de croisements plus nombreux et d'une sélection plus intense que le reste de la population d'amélioration, visant à obtenir un gain génétique rapide directement utilisable par les sylviculteurs. Le reste de la population constitue, quant à lui, la garantie d'une base génétique large.

\section{Intégration des marqueurs moléculaires dans le cadre de la réorganisation du schéma d'amélioration}

Dans ce contexte d'une restructuration du schéma d'amélioration du pin maritime, nous pouvons envisager l'utilisation des marqueurs moléculaires comme caractère associé au phénotype pour améliorer la sélection individuelle (sélection intrafamille analogue à une sélection massale multicaractère dans les familles retenues après l'étape de sélection familiale). Les marqueurs pourraient être utilisés à deux niveaux :
- Dès à présent pour constituer les populations élites. La sélection des individus $\mathrm{G} 1$ et $\mathrm{G} 2$ de la seconde et de la troisième population d'amélioration (Baradat et Pastuz$\mathrm{ka}, 1992)$ a été réalisée entre 8 et 10 ans dans des tests de descendance (familles «open " issues de la pollinisation libre en forêt et familles de plein-frères). L'âge de cette sélection a depuis peu été réévalué à la hausse ( $\geq 12$ ans, Kremer, 1992 ; Danjon, 1994) et les pondérations des caractères cibles de la sélection ont évolué, mettant de plus en plus l'accent sur la qualité (rectitude du fût, branchaison, infradensité du bois). Ainsi, les individus G1 et G2 seront prochainement l'objet d'une nouvelle sélection dans les tests d'au moins 12 ans. Ceci devrait permettre de garantir le maximum de gain génétique pour les variétés futures et d'accroître le niveau global de ces deux populations d'amélioration. Dans ce cadre, pour les familles possédant de grands effectifs, les marqueurs pourraient être intégrés dans un index de sélection combiné avec les caractères phénotypiques, afin de garantir le choix optimal des futurs géniteurs qui pourraient constituer les populations élites.

- Dans le futur, le repérage des meilleurs individus sur la base d'une information combinant le phénotype et les marqueurs pourrait être entrepris dans les meilleures familles de plein-frères à l'intérieur des sous-populations constituants la population principale. Les bons recombinants ainsi identifiés pourraient alors être utilisés pour réalimenter les populations élites ainsi que pour reconstituer les sous-populations de la génération suivante.

D'autre part, l'utilisation des marqueurs pourrait être encore plus développée dans le cadre de la création de variétés de pin maritime "à la carte " (variétés "vigueur ", " rectitude ", " résistance à la sécheresse "), optimisées pour des utilisations particulières et produites dans des vergers à graines spécialisés. En effet, la SAM pourrait permettre d'augmenter la fré- 
quence des allèles favorables aux QTL pour un caractère donné, rendant la sélection plus intense sur un caractère économiquement intéressant et à faible héritabilité (l'écart à la verticalité basale par exemple). II convient également de noter que les allèles au QTL ayant une fréquence faible dans la population ainsi qu'un effet favorable, devraient être les plus intéressantes pour accroître la valeur moyenne de la population d'amélioration (Plomion et Durel, 1996).

\section{Méthodologie de la sélection intrafamille assistée par marqueurs}

L'utilisation des marqueurs RAPD pour la sélection intrafamille et/ou la recherche des bons recombinants basée sur les marqueurs moléculaires, ainsi que la valorisation de la sélection en termes de création variétale, peut s'envisager selon un schéma en six étapes présenté à la figure 1 :

- Étape 1 : Les familles de plein-frères (FS) candidates à la SAM intrafamille doivent d'abord passer par un triple crible. Primo, comme nous l'avons indiqué précédemment, ces familles doivent avoir un effectif suffisant ( $\geq 200$ individus). Secondo, ces familles doivent avoir atteint l'âge de la sélection (12 à 15 ans). Tertio, les parents doivent avoir aussi été testés en polycross (voir étape 4). Dans l'état actuel du programme d'an iélioration, certaines familles

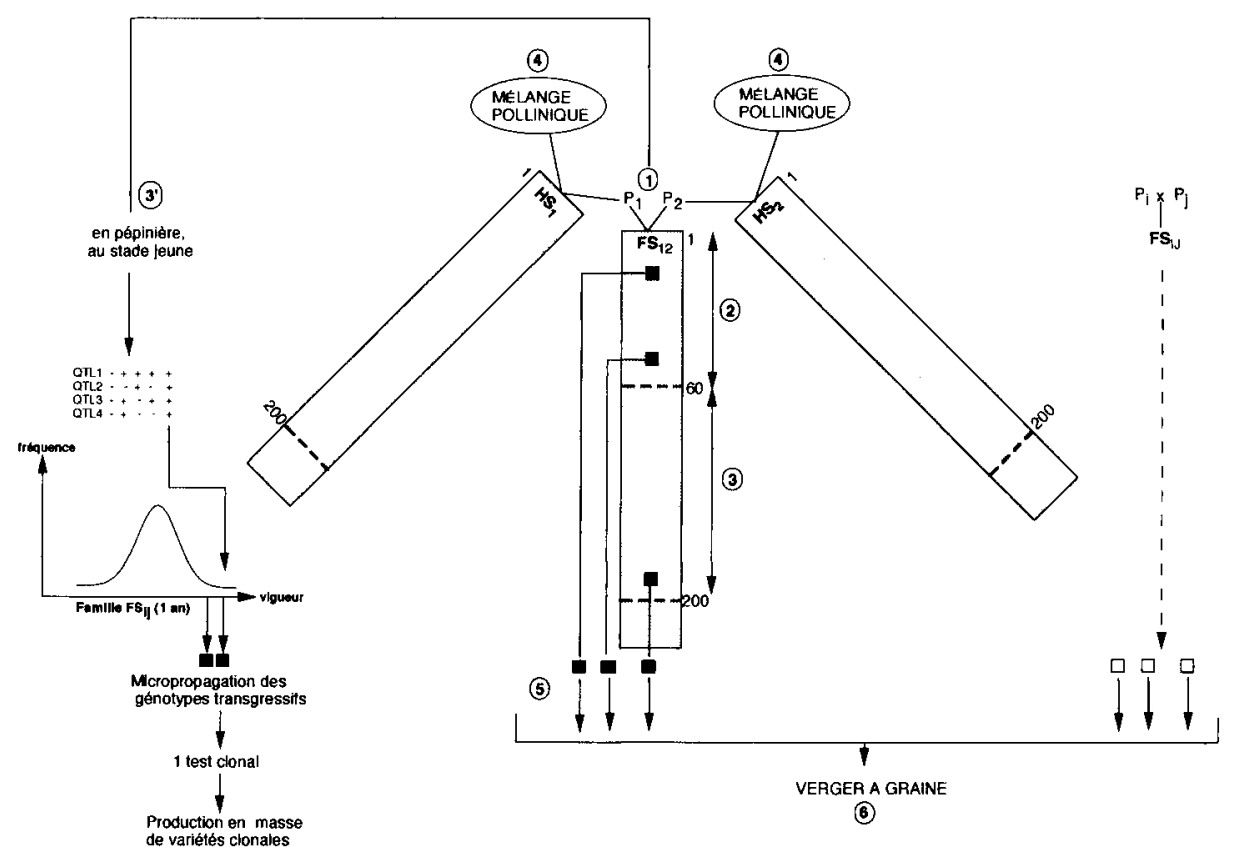

Fig 1. Étapes de la sélection intrafamille assistée par marqueurs, associant la multiplication végétative au stade juvénile. La cartographie génétique et la détection de QTL spécifiques sont envisagées dans des familles de plein-frères (FS) ; la généralisation des QTL spécifiques en QTL généraux se fait par l'intermédiaire des deux polycross (HS) issus des deux parents. Les chiffres entourés correspondent aux étapes décrites au paragraphe "Méthodologie de la sélection intrafamille assistée par marqueurs", les nombres 60 et 200 correspondent respectivement aux effectifs pour la cartographie et la détection de QTL. 
$\mathrm{G} 1$ et $\mathrm{G} 2$ répondent à ces deux critères. - Étape 2 : Construction de cartes génétiques à l'aide de la stratégie du "double pseudo-testcross » pour les parents impliqués dans les familles FS choisies dans la première étape. Un échantillon de 60 descendants permet une bonne estimation des taux de recombinaison. Les marqueurs RAPD seraient privilégiés pour les raisons indiquées précédemment.

- Étape 3 : Établissement des associations marqueurs-QTL spécifiques pour les caractères mesurés dans les tests. Les descendances devront être génotypées pour un sous-ensemble de marqueurs répartis environ tous les $20 \mathrm{cM}$ sur la carte génétique. Un nombre relativement important d'individus devrait être génotypé dans chaque famille (au moins 200 descendants), afin de détecter la plupart des QTL avec une puissance statistique suffisante (Darvasi et al, 1993)

- Étape 4 : Recherche de la valeur générale des QTL spécifiques. Chaque parent (P1 et P2) d'une famille FS doit être impliqué dans une famille de demi-frères. En effet, la stratégie de détection de QTL à partir d'une famille FS aboutit à la localisation de QTL “ spécifiques » à cette famille. Afin que ces QTL puissent être utilisables en sélection, il convient de rechercher leur valeur générale. La valeur additive des QTL spécifiques en situation hétérozygote chez l'un des parents peut être testée grâce aux familles polycross réalisées sur $\mathrm{P} 1$ et $\mathrm{P} 2$. De façon générale, si l'on suppose qu'il y a équilibre de liaison entre les allèles des marqueurs bordant le QTL et les allèles du QTL dans le mélange pollinique, le polycross faisant intervenir le parent chez lequel les marqueurs sont homozygotes nuls permet de calculer les fréquences alléliques des marqueurs bordant le QTL. L'autre polycross faisant intervenir le parent chez lequel les marqueurs sont à l'état hétérozygote permet de tester la valeur additive des QTL « spécifiques » connaissant la fréquence des marqueurs bordants (Plomion et Durel, 1996).

- Étape 5: À l'intérieur des familles FS, une sélection sur index combinant l'information des associations marqueurs-QTL généraux et celles des mesures phénotypiques permettrait de retenir les deux ou trois meilleurs génotypes par famille qui constitueront la population d'amélioration de la génération suivante (sous-populations ou élites). Il serait alors nécessaire de disposer d'effectifs importants (au moins 200 individus) au sein de ces familles FS, non plus pour des questions de puissance de test statistique, mais afin d'accroître l'intensité de sélection.

Dans le cas où l'on considère plutôt la recherche des meilleurs recombinants, un fort effectif sera également souhaitable afin d'augmenter la probabilité de détecter les quelques génotypes recombinants ayant le profil voulu aux marqueurs bordant les QTL généraux. En effet, compte tenu de cet effectif (200 descendants au minimum), nous pouvons déterminer la probabilité de trouver au moins un individu portant les QTA (Quantitative trait alleles) favorables parmi les individus présentant les allèles intéressants aux marqueurs bordant. Nous considérons, pour le calcul de cette probabilité, que les QTL sont indépendants (situés sur des chromosomes différents ou séparés par un taux de recombinaison d'au moins $50 \mathrm{cM}$, s'ils sont situés sur le même chromosome). La probabilité d'obtenir un individu portant un nombre $N$ de QTA favorables dépend de la taille des fragments contenant les QTL et elle est simplement égale au produit des probabilités individuelles. Si nous faisons l'hypothèse que le segment chromosomique intéressant contient deux intervalles de longueur $r_{1}$ et $r_{2}$, cette probabilité est égale à : $p=[0,5 \times$ $\left.\left(1-r_{1}\right) \times\left(1-r_{2}\right)\right]^{N}$. Les phases de liaison des marqueurs bordants le QTA sont connues et n'interviennent pas dans le calcul de cette probabilité. Nous présentons au tableau III les valeurs de $p$ pour des intervalles de confiance de 20 et $40 \mathrm{cM}$ autour 
de la position la plus probable du QTL, correspondant respectivement à des cartes génétiques de densité moyennes de 10 et $20 \mathrm{cM}$. Le cas le plus vraisemblable correspond à un intervalle de confiance de 40 $\mathrm{cM}$. C'est le niveau de précision généralement obtenu avec les effectifs utilisés, des effets de substitution standardisés égaux à $0,5^{\star} \sigma$, une erreur de type I de $1 \%$ et une puissance de détection de $60 \%$ (Mangin, communication personnelle). Dans ce cas, un effectif de 200 individus est une limite inférieure pour espérer trouver un génotype cumulant 4 à 5 QTA favorables en une génération. Si l'on recherche une combinaison de bons QTA pour deux caractères à la fois, la probabilité d'obtenir un recombinant favorable à plus de cinq QTL est proche de 0 (tableau III) mais la SAM restera toujours plus efficace que la sélection massale (Lande et Thompson, 1990).

- Étape 6: La sortie variétale pourrait être réalisée en verger à graines d'équivalentclones (Baradat, 1987) qui permettent une diffusion du progrès génétique basée sur les meilleurs descendants des clones élites. Ce type de verger polycross réalise un rapport performances/coût très supérieur à celui des vergers de clones d'individus élites. Une trentaine de génotypes sélectionnés dans une trentaine de familles non apparentées semble correspondre à un optimum permettant de minimiser l'effet défavorable des croisements entre apparentés dans la variété améliorée (Baradat,
1987). La sortie variétale pourrait également être réalisée en verger à graines de pollinisations contrôlées (Alazard, 1992 ; Carson et al, 1992).

\section{Stabilité des associations détectées au} cours des générations successives

La stratégie de sélection intrafamille devrait également s'avérer utile dans les générations suivantes. Si les associations marqueurs-QTL sont assez étroites, elles ne devraient pas être trop diluées au cours des générations. En effet, un déséquilibre de liaison $D_{T}$, est attendu entre deux marqueurs sélectivement neutres, distants de $r<1 / T$, où $T$ est le nombre de générations panmictiques, qui ont suivi l'hybridation initiale (Kimura et Otha, 1971). Le déséquilibre résiduel est alors $D_{T}=(1-r)^{\top} \times D_{0}$, et il diminuera d'autant plus vite que les locus seront éloignés. Par exemple : dans un programme d'amélioration après trois générations d'intercroisements, des marqueurs distants d'un taux de recombinaison de $30 \%$ devraient être efficaces pour détecter des associations marqueurs-QTL $\left(D_{T}=0,343^{\star} D_{0}\right)$. Ainsi, lorsque des liaisons très étroites sont établies entre marqueurs bordants et QTL, le déséquilibre de liaison initial, $D_{0}$, établi dans les populations d'amélioration par linkage physique à la génération $n$ devrait subsister malgré le phénomène de la recombinaison à la génération $n+1$.

De plus, il semble illusoire de pouvoir fixer tous les allèles favorables par croisement

Tableau III. Probabilités d'avoir un individu portant N QTA favorables, parmi ceux présentant les allèles intéressantes aux marqueurs flanquants le QTL, en fonction de la longueur du segment chromosomique contenant le QTL. Exemple de deux segments de longueurs égales $\mathrm{r} 1=\mathrm{r} 2$.

\begin{tabular}{|c|c|c|c|c|c|c|c|}
\hline \multirow[t]{2}{*}{$r 1=r 2$} & & \multicolumn{6}{|c|}{ Nombre de QTA favorables (N) } \\
\hline & & 1 & 2 & 3 & 4 & 5 & 10 \\
\hline $10 \mathrm{cM}$ & Probabilité & 0.405 & 0.164 & $6.64^{*} 10$ & $2.69^{*} 10^{-2}$ & $1.09^{*} 10^{-2}$ & $1.2^{*} 10^{-4}$ \\
\hline $20 \mathrm{cM}$ & Probabilité & 0.320 & 0.102 & $3.27^{\star} 10$ & $1.05^{\star} 10^{-2}$ & $0.33^{\star} 10^{-2}$ & $1.1^{*} 10^{-5}$ \\
\hline
\end{tabular}


en une seule génération et ce, même au niveau d'un seul caractère. Les allèles à effets forts devraient être fixés après une génération de SAM, laissant la place pour d'autres QTL dans les générations suivantes. De nouveaux allèles et de nouveaux QTL pourront également être introduits et recherchés à chaque génération.

\section{Gains génétiques et coûts de la sélection intrafamille assistée par marqueurs}

Le gain génétique attendu de la sélection intrafamille dans des descendances de plein-frères s'écrit : $\Delta G=i_{w f} \times h_{w f}^{2} \times \sigma_{w f}$ (où $i_{w f}$ est l'intensité de sélection intrafamille, $h^{2}$ wf est l'héritabilté intrafamille et $\sigma_{w f}$ est l'écart type phénotypique intrafamille de plein-frères). II s'écrit encore :

$$
\begin{gathered}
\Delta G=i_{w f} \times \frac{\frac{1 / 2 \times \sigma^{2} A}{\sigma^{2} w f} \times \sigma_{w t}}{\operatorname{avec} \sigma_{w t}=} \\
\frac{\sqrt{\frac{1}{2}} \sigma^{2}{ }_{A}+\frac{3}{4} \sigma^{2} D+\sigma^{2} E}{}
\end{gathered}
$$

Un cas concret d'espérance de gain génétique a été calculé pour la hauteur à 10 ans (moyenne de $6,81 \mathrm{~m}$ ), dans le test 2.44.10 (plan de croisement factoriel de 80 familles) du programme de sélection du pin maritime. Les variances suivantes ont été obtenues : $\sigma^{2}{ }_{A}=1669$; $\sigma^{2} D=198 ; \sigma_{E}^{2}=2901 ; \sigma^{2}{ }_{w f}=3884$. L'héritabilité individuelle était de $h^{2}=0,35$. L'estimation du gain génétique attendu pour une intensité de sélection de $i_{w f}=2,135$ ( 3 individus sélectionnés sur 70 ) est $\Delta G=8,2 \%$.

Pour une héritabilité intrafamille $h^{2}{ }_{w f}=0,21$ (calculée à partir des données du dispositif 2.44.10) et un pourcentage de la variation génétique expliquée par les QTL de $50 \%$, l'efficacité de la sélection associant l'information des marqueurs à celle du phénotype est de $E=1,63$ (valeur obtenue à partir de l'équation 1 de Strauss et al, 1992), ce qui donne une espérance de gain génétique de $\Delta \mathrm{G}_{(\mathrm{SAM})}=13,3 \%$, pour la sélection intrafamille assistée par marqueur, dans des descendances de 200 individus où l'on en sélectionnerait huit. L'espérance du gain génétique obtenu par la SAM intrafamille est donc supérieur de $5,1 \%$ à celui obtenu par la seule sélection phénotypique intrafamille.

Nous avons représenté à la figure 2 les coûts du marquage moléculaire en fonction du nombre de familles génotypées. Ce coût prend en compte le fonctionnement et la main d'œuvre pour toutes les étapes allant de l'extraction d'ADN à la saisie informatisée des données. Les sommes obtenues résultent du calcul présenté au tableau II. La sortie de variétés améliorées, réalisées dans des vergers à graines d'équivalentclones, nécessite le choix d'un nombre suffisant de géniteurs non-apparentés (environ 30 individus élites). La recherche du meilleur recombinant dans 30 familles FS sur la base des QTL " généraux " nécessitera un investissement d'environ 2,7 millions de francs (MF) sur cinq ans pour un technicien, main d'œuvre et fonctionnement compris (tableau II).

L'obtention d'un gain équivalent à la SAM par sélection intrafamille basée uniquement sur le phénotype nécessiterait d'appliquer une intensité de sélection très intense: $\quad i_{w r}=4,15$, soit un individu sélectionné par famille de 11800 individus ! Ce qui montre l'énorme avantage de la SAM dans le cas de la sélection intrafamille. Notons que, dans le cas particulier d'une sélection intrafamille, nous ne pouvons pas calculer l'efficacité relative du nombre de répétitions des unités testées à intensité de sélection constante comme l'a proposé Gallais (1993). En effet, l'héritabilité intrafamille ne peut pas être améliorée en augmentant l'effectif de chaque famille. II faut noter que cet investissement ne s'adressera pas à la détection de QTL pour un seul caractère. En effet, une fois le marquage effectué, d'autres QTL pourront être mis en évidence sur d'autres caractères. L'investissement sera donc d'autant plus rentabilisé que la sélection sera multivariable. 


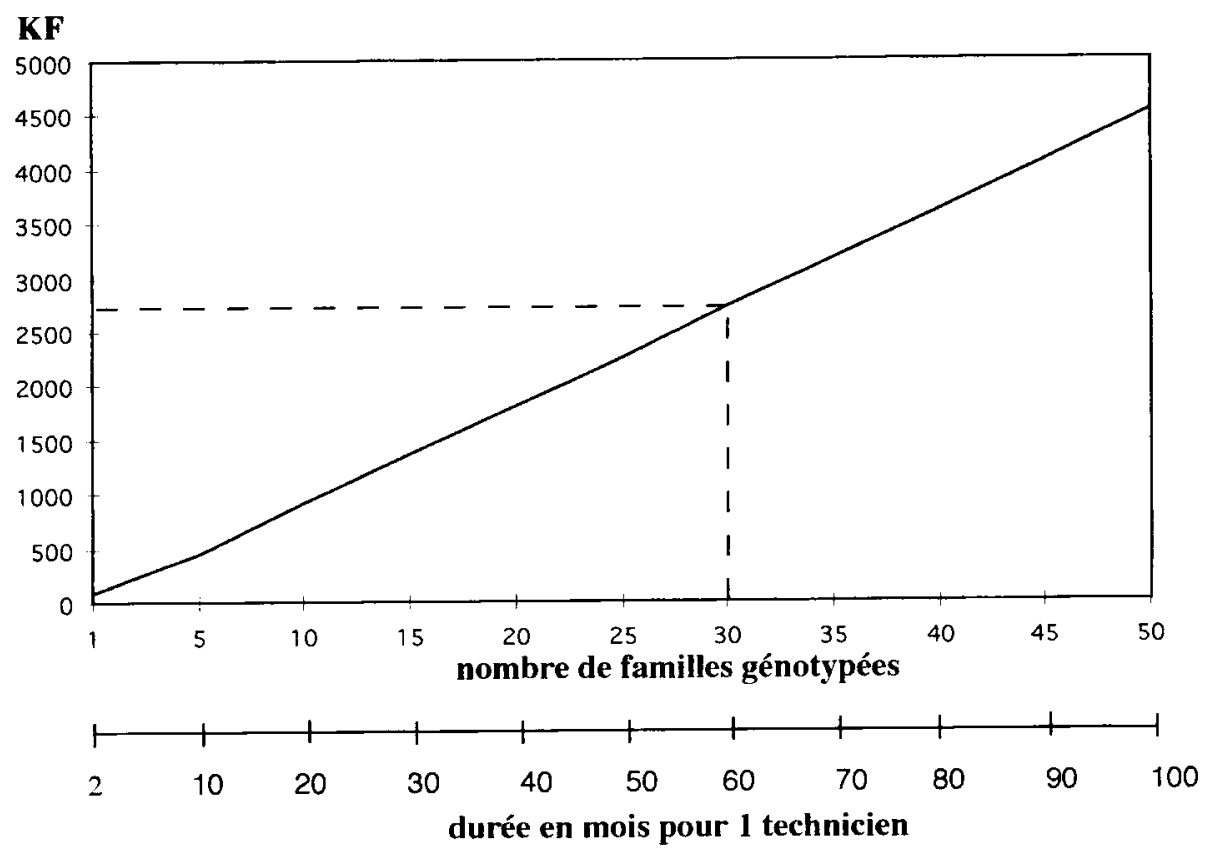

Fig 2. Coût (en KF) et durée (en mois) de la détection de QTL généraux à partir d'une famille de plein-frères et de deux familles de demi-frères. Calcul basé sur les données du tableau II.

Répercussion du coût de la SAM sur le coût de la graine améliorée

Le pin maritime couvre 1,4 million d'hectares en France. Il se situe en première position des reboisements français de conifères avec environ 20000 ha/an dont $50 \%$ par semis direct ( 10000 ha semés à raison de $1 \mathrm{~kg}$ de graines $/ \mathrm{ha}$ ) et $50 \%$ par plantation (10 000 ha plantés à raison de $1 \mathrm{~kg}$ de graines/12 ha). Dans le cas d'une période d'amortissement du coût du marquage moléculaire sur un cycle de sélection (12 ans), quel serait le surcoût de la graine issue de la SAM intrafamille par rapport à la graine améliorée (étiquette bleue) vendue actuellement à $1200 \mathrm{~F} / \mathrm{kg}$ ? Nous considérons pour ce calcul que la semence «SAM » sera plutôt destinée à la régénération par plantation, et que les 10000 ha reboisés annuellement par plantation le seront avec des variétés différentes (par exemple trois) ayant chacune une base génétique d'une trentaine de génotypes. Disposant de 10000 ha à reboiser par an et ce sur 12 ans, soit 120000 ha, il faudrait, à raison de $1 \mathrm{~kg} / 12 \mathrm{ha}$, produire $10000 \mathrm{~kg}$ de graines améliorées, soit $3333 \mathrm{~kg}$ par variété « SAM ».

Dans le cadre de la sélection assistée par marqueurs effectuée dès à présent (voir paragraphe «Intégration des marqueurs moléculaires dans le cadre de la réorganisation du schéma d'amélioration "), le surcoût de la graine "SAM » permettant d'amortir le coût du marquage moléculaire serait de $810 \mathrm{~F} / \mathrm{kg}(2,7 \mathrm{MF} / 3333 \mathrm{~kg})$ pour le typage de 30 familles. Comparé au prix du plant amélioré vendu par les pépiniéristes (de l'ordre de 1,50 F/plant), ce coût 
supplémentaire représenterait une augmentation du prix du plant d'environ 5,4 centimes

$$
\left(\frac{810 \mathrm{~F} / \mathrm{kg}}{15000 \text { plants } / \mathrm{kg}}\right)
$$

Dans le cas de la SAM appliquée dans le futur (paragraphe « Intégration des marqueurs moléculaires dans le cadre de la réorganisation du schéma d'amélioration »), il faut ajouter, au coût du marquage d'une trentaine de famille élites, les coûts de la sélection dans des familles d'au moins 200 individus. La sélection se fait actuellement sur des effectifs d'environ 70 individus. À titre d'exemple la mise en place d'un test de descendance de 100 familles de 200 individus représenterait un coût supplémentaire de 0,6 MF par rapport au coût actuel (calcul basé sur les données du tableau IV). Le surcoût de la graine améliorée par SAM serait alors de $990 \mathrm{~F}(2,7+$ $0,6 \mathrm{MF} / 3333 \mathrm{~kg}$ ), soit une augmentation du prix du plant d'environ 6,6 centimes.

Dans ces deux cas de figure, le prix du plant amélioré issu d'une sélection combinant l'information du phénotype et des marqueurs moléculaires devrait avoir une répercussion tolérable sur le prix du plant destiné à la plantation. Ainsi, il semble que la sélection intrafamille assistée par marqueurs soit avantageuse. Nous ne pouvons cependant pas prédire quels seraient les surcoûts répercutés par les pépiniéristes. L'espérance du gain génétique de la SAM intrafamille par rapport à la sélection phénotypique $(5,1 \%$ sur la hauteur soit environ $15 \%$ sur le volume, Danjon, 1995), représenterait un gain financier de $300 \mathrm{~F} / \mathrm{ha}$ an pour les sylviculteurs (calcul

Tableau IV. Coûts de la mise en place d'un test de descendance chez le pin maritime, de la préparation du terrain à la sélection des meilleurs individus à 12 ans. Le coût est calculé par hectare pour une densité à 12 ans de 1200 arbres/hectare.

\begin{tabular}{lll}
\hline Interventions & \multicolumn{1}{c}{ Étapes } & Coût (F/ha) \\
\hline Plantation & \begin{tabular}{l}
\multicolumn{1}{c}{ Élevage des plants en pépinière } \\
Désherbage ou préparation de la parcelle \\
\end{tabular} & 30000 \\
& $\begin{array}{l}\text { Fertilisation } \\
\text { Labour }\end{array}$ & \\
& Reprise du labour & \\
& Piquetage & \\
& Plantation (1 200 plants/ha)
\end{tabular}

Entretien Rotavator à 2 ans, 7000 gyrobroyage à 5 et 12 ans, élagage à $2 \mathrm{~m}$

Mensuration

$(1 \mathrm{HJ}=500 \mathrm{~F}=300$ mesures $=2000$ saisies $)$

15000

5 mesures : hauteur totale à 5 et 12 ans

circonférence à 5 et 12 ans

écart à la verticalité à 12 ans

Déplacement, saisie des données

Traitement (calcul d'index de sélection)

3000

des données

$1 \mathrm{HJ}=1500 \mathrm{~F}$ 
basé sur une production de $10 \mathrm{~m}^{3} / \mathrm{ha} / \mathrm{an}$ payée en moyenne $2000 \mathrm{~F}$ ).

\section{Sélection des géniteurs de variétés hybrides d'Eucalyptus, utilisant les marqueurs moléculaires}

La création de variétés hybrides de première génération entre espèces génétiquement éloignées et complémentaires (Eucalyptus urophylla et Eucalyptus grandis) est la solution choisie pour l'amélioration de l'Eucalyptus au Congo. Le schéma de sélection récurrente réciproque (SRR), développé depuis 1989, vise à améliorer de façon conjointe et orientée les deux espèces l'une par rapport à l'autre. Les géniteurs des hybrides sont sélectionnés sur leur $A G C$ en intercroisement, puis sont recombinés intrapopulation pour donner les populations de sélection au cycle suivant (Bouvet et al, 1992). À chaque génération, la complémentarité entre groupes devrait s'accentuer par l'augmentation de la fréquence des gènes favorables à l'intérieur de chaque population et la valeur attendue de l'hybride devrait augmenter (Gallais, 1978). Les familles hybrides servent au test des valeurs en croisement mais aussi à la sortie variétale où l'ASC est exploitée (Vigneron, 1991). La sortie variétale est réalisée sous forme clonale ou sous forme de familles de plein-frères reproduites par voie sexuée et/ou multipliées végétativement.

\section{Prédiction de l'hétérosis}

Chez les Eucalyptus comme chez de nombreuses espèces allogames, l'hétérosis peut être très fort, les meilleures familles hybrides présentent des performances de $25 \%$ supérieures aux meilleures espèces parentales (Vigneron, 1991). Néanmoins, la polygénie des caractères de croissance rend difficile la fixation de cet hétérosis qui résulte probablement d'une complémentarité allélique entre les deux espèces (vigueur hybride) plutôt que de la superdominance. L'utilisation des marqueurs moléculaires est envisagée pour optimiser le choix des meilleurs parents à croiser dans les différents plans de croisement. Dans un premier temps, les marqueurs sont utilisés pour calculer les distances génétiques entre les parents. Puis la recherche d'associations entre l'aptitude spécifique à la combinaison (ASC) des combinaisons hybrides ou l'aptitude générale à la combinaison (AGC) des parents avec les marqueurs devrait permettre de définir des segments chromosomiques spécifiques et/ou généraux intervenant potentiellement dans l'expression des caractères quantitatifs. À terme, la SRR entre $E$ urophylla et $E$ grandis rendra possible la fixation des allèles favorables complémentaires dans chaque espèce.

Les premiers résultats obtenus sur Eucalyptus ne mettent pas en évidence de relation entre l'hétérosis et les distances génétiques calculées à partir de l'ensemble des marqueurs ( 415 fragments RAPD, Verhaegen et al, 1995). Ces résultats expérimentaux sont conformes aux résultats théoriques qui montrent qu'il est peu probable que la prédiction au travers de la distance (calculée avec tous les marqueurs) entre parents n'ayant pas d'origine commune soit efficace (Charcosset et Essioux, 1994). II reste cependant plusieurs axes de recherche à explorer. Le premier concerne l'étude du déséquilibre de liaison entre marqueurs moléculaires et QTL : condition nécessaire pour trouver une relation entre hétérosis et hétérozygotie évaluée au niveau moléculaire (Charcosset et al, 1991). Ce point est vraisemblablement vérifié car les deux populations d'amélioration sont constituées d'un nombre réduit d'arbres sélectionnés dans des provenances très localisées. Les $E$ urophylla sont originaires d'une des îles de la Sonde, et les $E$ grandis proviennent de l'extrême nord de l'aire naturelle en Australie. L'isolement de ces provenances naturelles depuis des générations augmente la probabilité d'observer un déséquilibre de liaison entre marqueurs et QTL. II conviendra donc au préalable de 
quantifier le niveau de ce déséquilibre pour les marqueurs utilisés dans les deux populations d'amélioration. Les premiers résultats expérimentaux font apparaître des associations significatives pouvant être attribuées au déséquilibre de liaison entre marqueurs et caractères quantitatifs (Verhaegen et al. 1995). Un autre axe de recherche concerne la structuration des populations parentales servant à la création variétale. Ainsi, pour le maïs, la prédiction de l'hétérosis du rendement en grains a été mise en évidence à partir des coefficients de parenté révélés sur des lignées parentales par RFLP (Bernardo, 1994). Les fortes corrélations observées $(0,65$ à 0,80$)$ montrent que les performances des hybrides peuvent être effectivement prédites en utilisant l'information des hybrides existants.

Recherche des génotypes transgressifs dans des familles de plein-frères, combinée à la multiplication clonale

En raison du déséquilibre de liaison produit par l'hybridation et de l'importante variance phénotypique, les programmes d'hybridation interspécifique se prêtent mieux à la sélection assistée par marqueurs (Strauss et al, 1992). La recherche de génotypes cumulant les bons QTA pourrait être réalisée au stade adulte mais également à plus grande échelle au stade jeune en pépinière (étape 3', de la figure 1). Les croisements impliquant les parents ayant fourni les familles les plus intéressantes pourraient alors être refaits. La sélection des recombinants favorables s'effectuerait alors à l'aide des seuls marqueurs ayant montré une association avec les QTL spécifiques au stade adulte. Chez une espèce comme l'Eucalyptus pour laquelle la multiplication végétative est utilisée pour la création variétale, l'utilisation des QTL spécifiques serait optimale puisque les effets additifs et de dominance seraient exploités. La sélection des meilleurs recombinants sur la base des marqueurs moléculaires et du phénotype, combinée à leur multiplication végétative devrait permettre la confirmation des meilleurs génotypes en une seule série de test clonal, alors que deux séries sont actuellement nécessaires (Delwaulle, 1988). Une sortie variétale considérablement accélérée, grâce à une forte réduction de la durée d'évaluation des clones, augmentera le gain génétique par unité de temps, entraînant une baisse des coûts d'installation en plantations industrielles et une augmentation des revenus.

\section{Estimations des gains génétiques et des coûts de la sélection assistée par marqueurs}

Nous nous plaçons dans le cas d'une sélection combinée individu-famille. Les progrès génétiques attendus ont été calculés pour une sélection basée sur la seule information phénotypique ainsi que pour la sélection assistée par marqueurs selon l'équation [1] de Strauss et al (1992). Afin de prendre en compte le coût d'acquisition des données des marqueurs moléculaires, nous avons modifié cette équation en y intégrant la proportion (s) de familles génotypées après l'étape de sélection familliale. L'efficacité s'écrit alors :

$$
\begin{gathered}
E=\frac{h_{f}+(1-s) \times \frac{i_{w f}}{i_{f}} \times h_{w f}+}{h_{f}+\frac{i_{w f}}{i_{f}} \times h_{w f}} \\
\frac{s \times \frac{i_{w f}}{i_{f}} \times \sqrt{\frac{p+(1-p)^{2} \times h^{2}{ }^{2} f}{1-\left(h^{2}{ }^{2} \times p\right)}}}{h_{f}+\frac{i_{w f}}{i_{f}} \times h_{w f}}
\end{gathered}
$$

Un cas concret d'espérance de gain génétique a été calculé pour la circonférence à 3 ans et demi, dans le test R90-11 (plan de croisement factoriel incomplet équilibré de 94 familles) du programme d'amélioration. Ce caractère a été retenu, car il est bien corrélé avec la production des arbres en fin de rotation. Avec une sélection inter- 


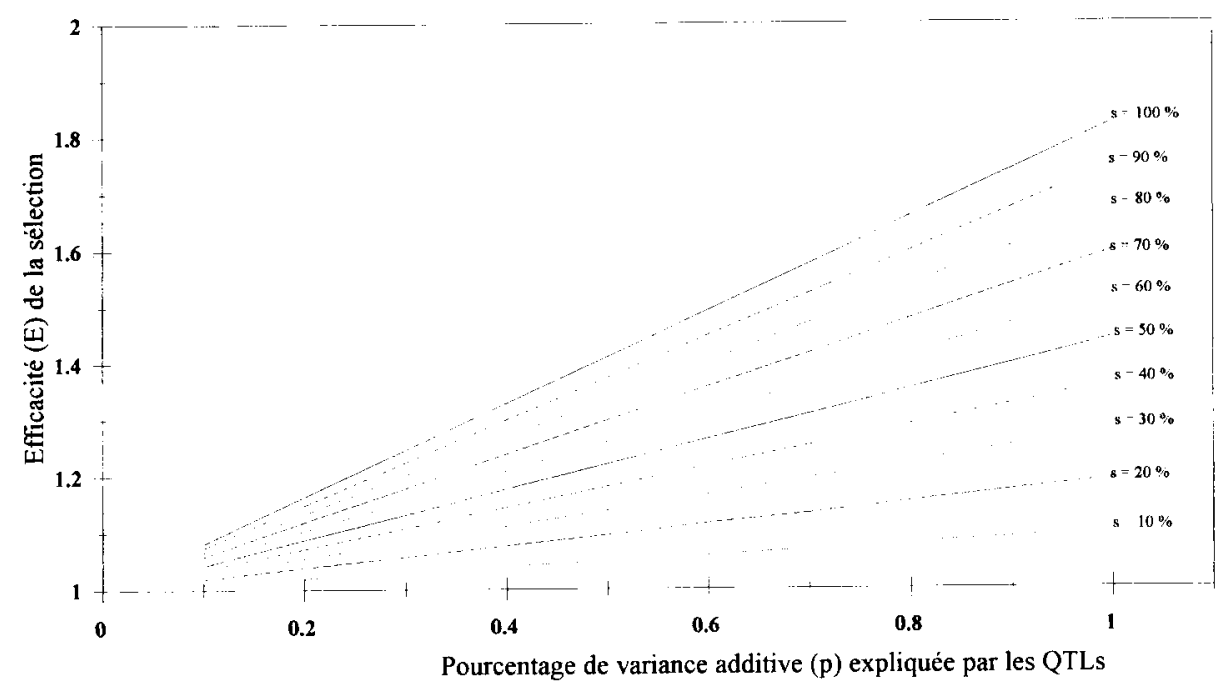

Fig 3. Efficacité (E) de la sélection assistée par marqueurs (cas de la sélection combinée individufamille), par rapport à la sélection phénotypique seule pour la circonférence 3 ans et demi, en fonction de la proportion de variance additive $(p)$ expliquée par les QTL. $i_{f}=0,798$ (intensité inter-famille), $i_{w f}$ $=2,502$ (intensité intrafamille), $h^{2}=0,47$ (héritabilité inter-famille) et $h_{w t}^{2}=0,2$ (héritabilité intrafamille), $s$ (proportion des familles génotypées).

famille peu intense $\left(i_{f}=0,798\right.$, soit la moitié des familles retenues), une sélection individuelle forte $\left(i_{w f}=2,502\right.$, soit un clone sélectionné par famille de 64 plants), une héritabilité intrafamille $h^{2} w f=0,20$ et une héritabilité interfamille $h_{f}^{2}=0,47$, une espérance de gain génétique de $3,8 \%$ est attendue pour la sélection phénotypique.

Pour un pourcentage de la variation génétique expliquée par les QTL de $50 \%$ $(p=0,50)$ et une proportion des familles génotypées de $40 \%(s=0,40)$, l'efficacité de la sélection associant l'information des marqueurs est de $E=1,17$ (fig 3 ) ; faisant ainsi passer le progrès génétique à $4,4 \%$. En se référant aux calculs des coûts présentés au tableau II, la cartographie ainsi que que la détection des QTL spécifiques dans $40 \%$ des familles (soit 19 sur 47 familles retenues) est estimé à environ $1164000 \mathrm{~F}$. La mise en place d'un nombre plus important d'individus par famille de plein-frères a pour effet d'augmenter $h^{2}$. Cependant, comme dans l'exemple de la sélection intrafamille chez le pin maritime, il faudrait un très grand nombre de répétitions pour atteindre une efficacité comparable. Une efficacité maximale de $E=1,44$ devrait être atteinte pour $\mathrm{s}=100 \%$ des familles typées (soit 47) pour un coût de $2900000 \mathrm{~F}$.

Actuellement, la confirmation de la supériorité des clones nécessite la mise en place de deux séries de tests clonaux réalisés sur 14 ans. La stratégie utilisant l'information conjointe des marqueurs et du phénotype devrait permettre d'identifier plus précisément les meilleurs génotypes et ainsi de réduire à un test clonal l'évaluation des clones, soit un gain d'environ 5 ans dans le schéma d'amélioration génétique de l'eucalyptus. Le repérage rapide des individus transgressifs devrait permettre 
d'augmenter les gains génétiques par unité de temps par rapport au schéma actuel.

\section{Multiplication végétative assistée par marqueurs}

L'utilisation des marqueurs pour la sélection des parents des futures générations (SAM) est à distinguer de leur utilisation pour la sélection d'individus candidats au déploiement des variétés clonales. Cette distinction est importante et a souvent été occultée dans la littérature, puisque la multiplication végétative ne présente pas d'intérêt chez la plupart des plantes agronomiques annuelles. La propagation par voie végétative est très utilisée chez les arbres forestiers (Eucalyptus, Populus, Pinus radiata, hybrides entre Pinus caribaea et $P$ inus elliotii...) et est certainement le moyen le plus efficace de valoriser l'information apportée par les marqueurs. La forestrie clonale implique le déploiement d'un petit nombre de variétés testées répondant à une demande précise (variétés "à la carte ") et permettant un accroissement de l'efficacité lors de l'exploitation (Libby, 1992). Libby et Rauter (1984) ont présenté les principaux avantages de ce type de variétés et, durant ces 10 dernières années, l'intérêt pour la multiplication clonale s'est considérablement accru (Kleinschmidt et al, 1993 ; Gupta et al, 1993 ; Talbert et al, 1993). Nous avons précédemment montré que la recherche de QTL spécifiques était économiquement réalisable dans un petit nombre de familles. Dans ce cadre, la SAM intrafamille qui vise à l'identification de l'idéotype au sein de familles de pleinfrères en utilisant l'information du phénotype et des marqueurs liés aux QTL des caractères d'intérêt économique devrait avoir une place importante. Le maintien d'une base génétique large au sein des populations d'amélioration sera le principal garant d'une diversité constamment renouvelée au niveau des variétés clonales. Les marqueurs pourront également avoir un rôle important dans la gestion des populations d'amélioration.

\section{CONCLUSION}

Dans une revue portant sur l'utilisation potentielle de la SAM pour l'amélioration des arbres forestiers, Strauss et al (1992) concluaient à une utilisation plutôt limitée des marqueurs moléculaires. L'argumentation reposait essentiellement sur deux points : le faible déséquilibre de liaison rencontré chez la plupart des espèces forestières et les coûts (non chiffrés) du typage moléculaire. Depuis, les techniques de marquage ont évolué, permettant la construction de nombreuses cartes génétiques et une détection plus rapide des QTL. À la lumière de ces nouveaux résultats, nous avons reconsidéré les arguments développés par Strauss et al (1992) et cherché à évaluer l'intérêt potentiel de l'utilisation des marqueurs RAPD dans les programmes d'amélioration du pin maritime et de l'eucalyptus. L'avantage principal des marqueurs RAPD réside dans la simplicité de mise en œuvre et d'utilisation de la technique ainsi que dans la possibilité d'exécuter rapidement les milliers de réactions nécessaires à la cartographie et à la détection de QTL à grande échelle. Par ailleurs, ces marqueurs ne nécessitent pas d'information a priori de séquences nucléotidiques, ce qui permet de les utiliser chez des espèces dont le génome est peu caractérisé. Des cartes génétiques peuvent alors être construites pour de nombreux géniteurs et des associations entre marqueurs et caractères quantitatifs établies à l'intérieur de chaque famille, permettant ainsi de s'affranchir du faible déséquilibre de liaison existant au niveau de la population.

Nous avons montré qu'un progrès génétique supérieur à celui obtenu par les méthodes de sélections classiques pouvait résulter de la sélection de génotypes transgressifs basées sur l'information phé- 
notypique et moléculaire. Cependant, l'avenir de la SAM dépendra des coûts supplémentaires du marquage moléculaire et des retours sur investissements en termes de gain génétique. Dans l'immédiat, la technique de marquage RAPD permet d'envisager l'utilisation de ces marqueurs dans le cadre de la sélection intrafamille et de la sélection combinée individu-famille et ce, pour un nombre limité de familles.

Une autre utilisation intéressante des marqueurs moléculaires concerne la sélection indirecte de caractères difficiles et coûteux à évaluer (par exemple : rendement en pulpe, qualité du bois, capacité d'enracinement, résistance aux parasites et aux ravageurs). Enfin, les marqueurs constituent un moyen très efficace pour l'étude du déterminisme génétique de caractères complexes, en décomposant ceux-ci en éléments plus simples suceptibles d'être contrôlés par quelques gènes à effets majeurs et manipulables par le sélectionneur.

Pour ce qui est de la méthodologie que nous avons développée ici, nous n'avons pas envisagé la possibilité d'analyser plusieurs familles et de fusionner les cartes individuelles pour les parents des familles FS. Moyennant un plan de croisement approprié (diallèle, factoriel), il devrait être possible de connecter de proche en proche les différentes cartes pour établir une carte consensus. L'obtention d'une telle carte permettrait de rechercher les QTL en analysant simultanément plusieurs descendances, ce qui augmenterait la puissance de détection des QTL (Maranty, 1996). La connexion des cartes permettrait d'envisager des méthodes de sélection assistée par marqueurs comme celles développées en génétique animale (Fernando et Grossman, 1989) sur des structures de pedigree voisines des familles utilisées dans les programmes de sélection des espèces forestières. Cependant, il n'est pas évident que des marqueurs bialléliques comme les RAPD puissent convenir dans ce cadre. Le développement de marqueurs codominants et multialléliques comme les microsatellites semble plus adapté pour le développement d'une telle stratégie. Cependant, ce type de marqueur n'est pratiquement pas développé chez les arbres forestiers.

Les marqueurs moléculaires constituent un outil supplémentaire mis à la disposition du sélectionneur. En combinaison avec les analyses issues de la génétique quantitative, les techniques de multiplication végétative et la gestion raisonnée des plantations, ils devraient pouvoir jouer un rôle, d'une part dans l'identification et la caractérisation des meilleurs recombinants permettant ainsi de constituer les nouvelles populations d'amélioration, et d'autre part pour le déploiement de variétés aux performances accrues.

\section{REMERCIEMENTS}

Nous remercions vivement A Kremer pour ses conseils lors de la rédaction du manuscrit, ainsi que JM Bouvet, $P$ Vigneron et $Y$ Hervé et les lecteurs pour leurs remarques de fond.

\section{RÉFÉRENCES}

Alazard P (1992) Production de variétés améliorées en verger de pollinisation contrôlée chez le pin maritime. In : Afocel/UFRO Symp "Mass production technology for genetically improved fast growing forest tree species ", Bordeaux, France, tome 2, 27-34

Allard R (1956) Formulas and tables to facilitate the calculation of recombination values in heredity. Hilgardia 24, 235-278

Avery PJ, Hill WG (1979) Distribution of linkage disequilibrium with selection and finite population size. $\mathrm{Ge}$ net Res (Camb) 33, 29-48

Bahrman N, Damerval C (1989) Linkage relationships of loci controlling protein amounts in maritime pine (Pinus pinaster Ait). Heredity 63, 267-274

Baradat $P$ (1987) Méthodes d'évaluation de la consanguinité chez les plants issus des vergers à graines de semis de première génération. III. Application à l'optimisation d'une alternative économique aux vergers à graines de clones d'élites : les vergers d'équivalents-clones. Silvae Genetica 36, 134-144 
Baradat P, Pastuzka P (1992) Le pin maritime. In : Amélioration des espèces végétales cultivées (A Gallais, H Bannerot, eds), Inra, Paris, 695-709

Beavis WD, Grant D, Albertsen M, Fincher R (1991) Quantitative trait loci for plant height in four maize populations and their associations with qualitative genetic loci. Theor Appl Genet 83, 141-145

Beckman JS, Soller M (1983) Restriction fragment length polymorphisms in genetic improvement: methodologies, mapping and costs. Theor Appl Genet $67,35-43$

Beckman JS, Soller M (1986) Restriction fragment length polymorphisms in genetic improvement of agricultural species. Euphytica 35, 111-124

Beever JE, George PD, Fernando RL, Stormont CJ, Lewin HA (1990) Associations between genetic markers and growth and carcass traits in a paternal halfsib family of angus cattle. J Anim Sci $68,337-344$

Bernardo R (1994) Prédiction of maize single-cross performance using RFLPs and information from related hybrids. Crop Sci 34, 20-25

Binelli G, Bucci G (1994) A genetic linkage map of Picea abies Karts, based on RAPD markers, as a tool in population genetics. Theor App/ Genet 88, 283-288

Botstein D, White RL, Skolnick M, Davis RW (1980) Construction of a genetic linkage map in man using restriction fragment length polymorphisms. $A m \mathrm{~J}$ Hum Genet 32, 314-331

Bouvet JM (1991) Geno-phenotypic regression and juvenile mature correlations: methodological tools for clonal selection of Eucalyptus hybrids in Congo. In : IUFRO Symp Intensive Forestry: the role of Eucalypts, Durban, South Africa, 13-18

Bouvet JM (1992) A new approach for clonal test in Congo. In : Afocel/IUFRO Symp Mass production technology for genetically improved fast growing forest tree species, Bordeaux, France, tome 1, 339347

Bouvet JM, Couteau N, Vigneron P (1992) Premiers éléments de l'analyse des plans factoriels du schéma de sélection récurrente réciproque de l'eucalyptus au Congo. in : Afocel/IUFRO Symp Mass production technology for genetically improved fast growing forest tree species, Bordeaux. France, tome 1, 317-326

Bouvet JM, Vigneron P (1995) Age trends in variances and heritabilities in Eucalyptus factorial mating designs. Silvae Genetica 44, 206-216

Bradshaw HD, Foster GS (1992) Marker-aided selection and propagation systems in trees: advantages of cloning for studying quantitative inheritance. Can J For Res 22, 1044-1049

Bradshaw HD, Villar M, Watson BD, Otto KG, Stewart $S$, Stettler RF (1994) Molecular genetics of growth and development in Populus. III. A genetic linkage map of a hybrid poplar composed of RFLP, STS, and RAPD markers. Theor Appl Genet 89, 167-178

Bradshaw HD, Stettler RF (1995) Molecular genetics of growth and development in Populus. IV. Mapping QTLs with large effects on growth, form and phenology traits in a forest tree. Genetics 139, 963-973
Byrne M, Murrell JC, Allen B, Moran GF (1995) An integrated genetic linkage map for eucalyptus using RFLP, RAPD and isozyme markers. Theor App/ Genet $91,869-875$

Cai Q, Guy CL, Moore GA (1994) Extension of the linkage map in Citrus using random amplified polymorphic DNA (RAPD) markers and RFLP mapping of cold-acclimatation-responsive loci. Theor Appl genet $89,606-614$

Carlson JE, Tulsieram LK, Glaubitz JC, Luk VWK, Kauffeld C, Rutledge R (1991) Segregation of random amplified DNA markers in F1 progeny of conifers. Theor Appl Genet 83, 194-200

Carson MJ, Vincent TG, Firth A (1992) Control-pollinated and meadow seed orchards of radiata pine. In : Afocel/UFRO Symp Mass production technology for genetically improved fast growing torest tree species, Bordeaux, France, tome 2, 13-20

Charcosset A, Essioux L (1994) The effect of population structure on the relationship between heterosis and heterozygosity at marker loci. Theor Appl Genet 89 , 336-343

Charcosset A, Lefort-Buson M, Gallais A (1991) Relationship between heterosis and heterozygosity at marker loci: a theoretical computation. Theor Appl Genet 81, 571-575

Conkle MT (1981) Isozyme variation and linkage in six conifer species. In : Proc Symp Isozymes of North American Forest Trees and Forest Insects. USDA For Serv Gen Tech Rep, PSW 48, 11-17

Cornelius $J$ (1993) Heritabilities and additive genetic coefficients of variation in forest trees. Can J ForRes 24, 372-379

Cotteril PP, Dean CA (1988) Changes in the genetic control of growth of radiata pine to 16 years and efficiencies of early selection. Sivae Genet 37, 138146

Danjon F (1994) Heritabilities and genetic correlations for estimated growth curve parameters in maritime pine. Theor App/ Genet 89, 911-921

Danjon F (1995) Observed selection effects on height growth, diameter and stem form in maritime pine. Silvae Genet 44, 10-19

Darvasi A, Weinreb A, Minke V, Weller JI, Soller (1993) Detecting marker-QTL linkage and estimating QTL gene effect and map location using saturated genetic map. Genetics 134, 943-951

Delwaulle JC (1988) Plantations clonales au Congo : point des recherches sur le choix des clones dix ans après les premières plantations. In : Proc IUFRO International Conference Breeding Tropical Trees, Section 2.02-08 Pattaya, Thailand

Devey ME, Fiddler TA, Liu BH, Knapp SJ, Neale BD (1994) An RFLP linkage map for loblolly pine based on a three-generation outbred pedigree. Theor Appl Genet 88, 273-278

Doyle JJ, Doyle JL (1987) Isolation of DNA from fresh plant tissue. Focus 12, 13-15

Dudley JW (1993) Molecular markers in plant improvement: manipulation of genes affecting quantitative traits. Crop Sci 33, 660-668 
Durel CÉ (1992) Gains génétiques attendus après sélection sur index en seconde génération d'amélioration du pin maritime. Rev For Fr 4, 341-355

Durel CÉ (1990) Paramètres génétiques et sélection en deuxième génération d'amélioration du pin maritime Pinus pinaster Ait. Thèse, INA Paris-Grignon, $205 p$

Echt CS, Kidwell KK, Knapp SJ, Osborn TC, McCoy TJ (1994) Linkage mapping in diploid alfalfa (Medicago sativa). Genome 37, 61-71

Edwards M (1992) Use of molecular markers in the evaluation and introgression of genetic diversity for quantitative traits. Field Crops Reseach 29, 241-260

van Eck HJ, Jacobs JME, Stam P, Ton J, Stiekema WJ, Jacobsen $E$ (1994) Multiple alleles for tuber shape in diploid potato detected by qualitative and quantitative genetic analysis using RFLPs. Genetics 137 , 303-309

Fernando RL, Grossman M (1989) Marker assisted selection using best linear unbiased prediction. Genet Sel Evol 21, 467-477

Furmier GR, Knowles P, Alesiuk MA, Dancik BP (1986) Inheritance and linkage of allozymes in seed tissue of whitebark pine. Can J Genet Cytol 28, 601-604

Gallais A (1978) Amélioration des populations, méthodes de sélection et création de variétés. Ill. Bases théoriques pour l'étude de la sélection récurrente réciproque. Ann Amélior Plantes 28, 637-666

Gallais A (1993) La sélection assistée par marqueurs. Le Sélectionneur Français 43, 43-62

Gerber S, Rodolphe F, Bahrman N, Baradat P (1993) Seed-protein variation in maritime pine ( $P$ inus pinaster Ait) revealed by two-dimensional electrophoresis: genetic determinism and construction of a linkage map. Theor Appl Genet 85, 521-528

Gradiner JM, Coe EH, Melia-Hancock S, Hoisington DA, Chao S (1993) Development of a core RFLP map in maize using an immortalized $\mathrm{F} 2$ population. Genetics 134, 917-930

Graef GL, Fehr WR, Cianzo SR (1989) Relation of isozyme genotype to quantitative characters in soybean. Crop Sci 29, 683-688

Grattapaglia D, Sederoft R (1994) Genetic linkage maps of Eucalyptus grandis and E urophylla using a pseudo-test cross mapping strategy and RAPD markers. Genetics 137, 1121-1137

Grattapaglia D, Bertolucci FL, Sederoff RR (1995) Genetic mapping of QTLs controlling vegetative propagation in Eucalyptus grandis and E urophylla using a pseudo-test cross mapping strategy and RAPD markers. Theor Appl Genet 90, 933-947

Grattapaglia D, Bertolucci FLG, Penchel R, Sederoff RR (1996) Genetic mapping of quantitative trait loci (QTLs) controlling growth and wood quality traits in Eucalyptus grandis using a half-sib family and RAPD markers. Genetics (sous presse)

Greenwood M, Hopper CA, Hutchison KW (1989) Maturation in Larch. I. Effect of age on shoot growth, foliar characteristics and DNA methylation. Plant Physiol 90, 406-412

Griffing AR, Cotterill PP (1988) Genetic variation in growth of outcrossed selfed and open pollinated pro- genies of Eucalyptus regnans and some implications for breeding strategy. Sivae Genetica 37, 124131

Groover A, Devey M, Fiddler T, Lee J, Megraw R, Mitchell-Olds T, Sherman B, Vujcic S, Williams C, Neale D (1994) Identification of quantitative trait loci influencing wood-specific gravity in an outbred pedigree of loblolly pine. Genetics $138,1293-1300$

Gupta PK, Pullman G, Timmis R, Kreitinger M, Carlson WC, Grob J, Welty E (1993) Forestry in the 21st century: the biotechnology of somatic embryogenesis. BioTechnology 11, 454-459

Haley CS (1991) Use of DNA fingerprints for the detection of major genes for quantitative traits in domestic species. Anim Genet 22, 259-277

Hamrick JL, Godt MJW, Scherman-Brayles ST (1992) Factors influencing levels of genetic diversity in woody plant species. New Forest 6, 95-124

Han K-H, Bradshaw HD, Gordon MP (1994) Adventitious root and shoot regeneration in vitro is under major gene control in an F2 family of hybrid poplar (Populus trichocarpax $P$ deltoïdes). Forest Genetics $1,139-146$

Hastings A (1989) The interaction between selection and linkage in plant populations. In : Plant Population Genetics, Breeding and Genetic Resources (Brown, Clegg, Kahler, Weir, eds), Sinauer associates Inc, Sunderland, MA, États-Unis, 163-180

Hayes PM, Liu BH, Knapp SJ, Chen F, Jones B, Blake T, Franckowiak J, Rasmusson D, Sorrells $M$, Ulirich SE, Wesenberg D, Kleinhofs A (1993) Quantitative trait locus effects and environmental interaction in a sample of North American barley germplasm. Theor Appl Genet 87, 392-401

Hospital F, Chevalet C, Mulsant P (1992) Using markers in gene introgression breeding programs. Genetics 132, 1199-1210

Hutchison K, Singer P, Volkaert $H_{1}$ Deker A, Costello L, Greenwood M (1994) A PCR-based molecular genetic map for the conifer Larix decidua. In : Abstract of the Second International Conference on the Plant Genome, San Diego, CA, États-Unis, abstract 90

Illy G (1966) Recherches sur l'amélioration génétique du pin maritime. Ann Sci For 23, 757-948

Kesseli RV, Paran I, Michelmore RW (1994) Analysis of a detailed genetic linkage map of Lactuca sativa (Lettuce) constructed from RFLP and RAPD markers. Genetics 136, 1435-1446

Kimura M, Otha T (1971) Theoretical aspects of population genetics. Princeton University Press, Princeton, NJ, États-Unis

Kleinschmidt J, Khurana DK, Gerhold HD, Libby WL (1993) Past, present, and anticipated applications of clonal forestry. In : Clonal forestry II: Conservation and applications (MR Ahuja, WJ Libby, eds), Springer-Verlag, Berlin, Allemagne, 9-41

Knapp SJ, Bridges WC (1990) Using molecular markers to estimate quantitative trait loci parameters: power and genetic variances for unreplicated and replicated progenies. Genetics 126, 796-777 
Kremer A (1992) Décomposition de la croissance en hauteur du pin maritime (pinus pinaster Ait) : architecture génétique et application à la sélection précoce. Thèse, université Paris-XI, $124 \mathrm{p}$

Kremer A (1994) Diversité génétique et variabilité des caractères phénotypiques chez les arbres forestiers. Genet Sel Evol 26, 105s-123s

Kremer A, Lascoux M, N'Guyen A (1991) Morphogenetic subdivision of height growth and early selection in maritime pine. In : Proc 21st Southern Forest Tree Improvement Conference, Knoxville, TN, ÉtatsUnis, 203-221

Lande R, Thompson R (1990) Efficiency of marker-assisted selection in the improvement of quantitative traits. Genetics 124, 743-756

Leonards-Schippers C, Gieffers W, Schäfer-Pregl R, Ritter E, Knapp SJ, Salamini F, Gebhardt C (1994) Quantitative resistance to Phytophtora infestans in potato: a case study for QTL mapping in an allogamous plant species. Genetics 137, 67-77

Libby WJ, Rauter RM (1984) Advantages of clonal torestry. For Chronicle 60, 145-149

Libby WJ (1992) Use of genetic variation for breeding forest trees. In : Plant Breeding in the 1990s (HT Stalker, JP Murphy, eds), 101-117

Liu BH, Hayes P (1993) Efficiency of GMAPB (genomeassisted plant breeding). In : Abstract of the First International Conference on the Plant Genome, San Diego, CA, abstract 85

Liu BH, Sederoff R, O'Maliey D, Whetten R (1994) Genome map-assisted plant breeding for forest trees. In : Abstract of the Second International Conference on the Plant Genome, San Diego, CA, États-Unis, abstract $F 11$

Liu BH, Sederoff R, O'Malley D (1995) Linkage analysis using open-pollinated populations. In : Proc 22st Southern Forest Tree Improvement Conference, Atlanta, GA, États-Unis, 489

McKeand SE (1988) Optimum age for family selection for growth in genetic tests of loblolly pine. For Sci $34,400-411$

McKeand SE, Bridgwater FE (1992) Third-generation breeding strategy for the North Carolina State University-industry cooperative tree improvement program. In : Proc IUFRO International Conference Breeding tropical trees, Section 2.02-08, Cali, Colombia

Mullis K, Faloona $F$ (1987) Specific synthesis of DNA in vitro via a polymerase-catalysed chain reaction. $M e-$ thods Enzymol 55, 335-350

Muona O (1982) Population structure of forest trees. Silva Fennica 16, 107-114

Muranty H (1996) Power of tests for QTL detection using full-sib families in different schemes. Heredity (sous presse)

Nelson CD, Nance WL, Doudrick RL (1993) A partial genetic linkage map of slash pine (Pinus elliotti Englem var elliottii) based on random amplified polymorphic DNAs. Theor App/ Genet 87, 145-151

Nelson CD, Kubisiak TL, Stine M, Nance WL (1994) A genetic linkage map of longleaf pine (Pinus palustris
Mill) based on random amplified polymorphied DNAs. $J$ Hered $85,433-439$

Niebling CR, Johnson K, Gerhold HD (1987) Electrophoretic analysis of genetic linkage in Scots pine (Pinus syivestris L). Biochem Genet 25, 803-814

Ohri D, Koshoo, TN (1986) Genome size in gymnosperms. Pl Syst Evol 153, 119-131

O'Malley DM, Guries RP, Nordheim EV (1986) Linkage analysis for 18 enzyme loci in Pinus rigida Mill. Theor Appl Genet 72, 530-535

Paterson AH, Lander ES, Hewitt JD, Peterson S, Lincoln SE, Tanksley SD (1988) Resolution of quantitative traits into Mendelian factors using a complete linkage map of restriction fragment length polymorphisms. Nature 335, 721-726

Paterson AH, Damon S, Hewitt JD, Zamir D, Rabinowitch $\mathrm{HD}$, Lincoln SE, Lander ES, Tanksley SD (1991) Mendelian factors underlying quantitative traits in tomato: comparison across species, generations and environments. Genetics 127, 181-197

Pichot C (1993) Analyse de dispositifs par approches itératives prenant en compte les performances des plus proches voisins. agronomie 13, 109-119

Plomion C (1995) Cartographie et déterminisme génétique de la hauteur juvénile chez le pin maritime ( $P$ inus pinaster Ait) en condition de croissance accélérée. Thèse, École nationale agronomique de Rennes, $187 \mathrm{p}$

Plomion C, Bahrman N, Durel C-E, O'Malley DM (1995a) Genomic mapping in Pinus pinaster (maritime pine) using RAPD and protein markers. Heredity 74, 661-668

Plomion C, O'Malley DM, Durel C-E (1995b) Genomic analysis in maritime pine (pinus pinaster). Comparison of two RAPD maps using selfed and open-pollinated seeds of the same individual. Theor App/Genet $90,1028-1034$

Plomion C, Durel E (1996) Estimation of the breeding value of specific alleles detected by the pseudo-test cross QTL mapping strategy. Gen Sel Evol (sous presse)

Plomion C, Yani A, Marpeau A (1996). Genetic determinism of 83 -carene in maritime pine using random amplified polymorphic DNA (RAPD) markers. Genome (sous presse)

Ragot M, Hoisington DA (1993) Molecular markers for plant breeding: comparisons of RFLP and RAPD genotyping costs. Theor Appl Genet 86, 975-984

Ragot M, Biasiolli M, Delbut MF, Dell'orco A, Malgarini $L$, Thevenin P, Vernoy J, Vivant J, Zimmermann R, Gay $G$ (1995) Marker-assisted backcrossing: a practical example. In : Colloque Techniques et utilisations des marqueurs moléculaires (A Bervilié, M Tersac, eds), Montpellier, 45-56

Ritter E, Gebhardt C, Salimi F (1990) Estimation of recombination frequencies and construction of RFLP linkage maps in plants from crosses between heterozygous parents. Genetics 125, 645-654

Shiraishi S (1988) Linkage relationships among allozyme loci in Japanese black pine, Pinus thunbergii Parl. Silvae genetica 37, 60-66 
Shrimpton AE, Robertson A (1988) The isolation of polygenic factors controlling bristle score in Drosophila melanogaster. II. Distribution of third chromosome bristle effects within chromosome sections. Genetics 11, 445-459

Smith DN, Devey ME (1994) Occurrence and inheritance of microsatellites in pinus radiata. Genome 37 . 977-983

Sniezko RA, Zobel BJ (1988) Seedling height and diameter variation of various degrees of inbred and outcross progenies of loblolly pine. Silvae Genetica 37 , 50-60

Sorensen FC, Miles RS (1982) Inbreeding depression in height, height growth, and survival of douglas fir, ponderosa pine and noble fir to 10 years of age. Forest Sci 28, 283-292

Strauss SH, Lande R, Namkoong G (1992) Limitations of molecular-marker-aided selection in forest tree breeding. Can J For Res 22, 1050-1061

Stuber CW (1989) Marker based selection for quantitative traits. Proc XII Congress of EUCARPIA. Göttingen Vortr Pflanzenzuchtg 16, 31-49

Stuber CW (1992) Biochemical and molecular markers in plant breeding. In : Plant Breeding Reviews (JW Dudley, AR Hallauer, M Ryder, eds), Vol 9, John Wiley \& Sons Inc, 37-61

Stuber CW, Sisco PH (1993) Marker-facilitated transfer of QTL alleles between elite inbred lines and responses in hybrids. 46th annual corn and sorghum research conference

Stuber CW, Goodman MM, Moll RH (1982) Improvement of yield and ear number resulting from selection at allozyme loci in a maize population. $\mathrm{Crop} \mathrm{Sci}$ 22, $737-740$

Szmidt AE, Muona $O$ (1989) Linkage relationships of allozyme loci in Pinus sylvestris. Hereditas 11, 91-97

Talbert CB, Ritchie GA, Gupta P (1993) Conifer vegetative propagation: an overview from a commercialization perspective. In : Clonal forestry II: Conservation and applications (MR Ahuja, WJ Libby, eds), Springer-Verlag, Berlin, Allemagne, 145-181
Tanksley SD, Hewitt JD ( 1988$)$ Use of molecular markers in breeding for soluble solids in tomato: a reexamination. Theor Appl Genet 75, 811-823

Tulsieram LK, Glaubitz JC, Kiss G, Carlson JE (1992) Single tree genetic linkage analysis in conifers using haploid DNA from megagametophytes. BioTechnology 10, 686-690

Verhaegen D, Kremer A, Vigneron P (1995) Relationships between heterosis and molecular polymorphism in interspecific crosses of $E$ urophylla $\times E$ grandis. In : Proc CRC/UFRO Conference Eucalypt Plantations, Improving Fibre Yield and Quality, Hobart, Australia, 434-437

Vigneron $P$ (1991) Création et amélioration de variétés d'hybrides d'Eucalyptus au Congo. In : IUFRO Symp Intensive Forestry: the role of Eucalyptus, Durban, South Africa, 345-360

Wakamiya I, Newton RJ, Johnston JS, Price HJ (1993) Genome size and environmental factors in the genus pinus. Am J Bot 80, 1235-1241

Weller JI, Kashi Y, Soller M (1990) Power of « daughter " and " granddaughter " designs for mapping of quantitative traits in dairy cattle using genetic markers. J Dairy Sci 73, 2525-2532

White TL, Hodge GR, Powell GL (1993) An advancedgeneration tree improvement plan for slash pine in the southeastern United States. Silvae Genet 42. 359-371

Williams JGK, Kubelik AR, Livak KJ, Rafalski JA (1990) DNA polymorphisms amplified by arbitrary primers are useful as genetic markers. Nucleic Acids Research 18, 6531-6535

Williams JGK, Hanafey MK, Rafalski JA, Tingey SV (1993) Genetic analysis using random amplified polymorphic DNA markers. Methods Enzymol 218. 704-740

Young ND, Tanksley SD (1989) RFLP analysis of the size of chromosomal segments retained around the $T \mathrm{~m}-2$ locus of tomato during backcross breeding. Theor App/ Genet 77, 353-359

Zobel B, Talbert J (1984) Applied forest tree improvement. J Wiley, New York, $505 \mathrm{p}$ 